\title{
Two Fault-tolerant Control Problems for Multiple-integrator Networks
}

\author{
A. Locatelli, N. Schiavoni*† \\ Dipartimento di Elettronica, Informazione e Bioingegneria, Politecnico di Milano, Piazza L. da Vinci, 32 - 20133 \\ Milano, Italy
}

\begin{abstract}
SUMMARY
The paper considers a network of agents with multiple-integrator internal dynamics, which share partial information on their states according to an arbitrary topology. For this system, two control problems are addressed and solved. The first consists in assigning the dominant closed-loop poles. The second consists in achieving a specified consensus with arbitrarily fast dynamics. In both cases, the regulator is required to be decentralized and the controlled network has to result tolerant with respect to faults in the communication apparatuses of the agents. Copyright (c) 2014 John Wiley \& Sons, Ltd.
\end{abstract}

Received ...

KEY WORDS: Network control; fault-tolerant control; dominant-dynamics assignment; consensus; decentralized control

\section{INTRODUCTION}

Networks are systems composed of subsystems, called agents, which share information useful for control. The relevant literature is very vast, and mainly concentrates on the case where the agents are identical and have single- or multiple-integrator dynamics. Most of the papers tackle consensus, synchronization, flocking ans similar coordination problems (see, e.g., [1-27]). Other papers face stabilization and pole-placement [28-30].

Within this general context, we consider two problems for networks whose agents have a multipleintegrator dynamics. We assume that: (i) the states available for measure are those corresponding to one or more of the highest integration levels; (ii) the agents share pieces of information according to an arbitrary topology; (iii) the regulators are decentralized. Furthermore, we include some faulttolerance conditions in the statements.

The first problem concerns the assignment of the closed-loop dynamics. Specifically, we aim at designing a regulator able to place the dominant poles of the controlled network near prespecified locations. Moreover, we assume that faults may occur in the agents' communication apparatuses.

\footnotetext{
*Correspondence to: N. Schiavoni: Dipartimento di Elettronica, Informazione e Bioingegneria, Politecnico di Milano, Piazza L. da Vinci, 32 - 20133 Milano, Italy

†Email:nicola.schiavoni@polimi.it

Copyright (C) 2014 John Wiley \& Sons, Ltd.
}

Prepared using rncauth.cls [Version: 2010/03/27 v2.00] 
In particular, we consider the possibility that: (i) one or more agents become unable to transmit information to other agents; (ii) one or more agents become unable to receive information from the other agents. We require that the dominant poles remain close to the desired positions in all these events.

We have already faced similar problems in [31-34]. These papers make reference to the stabilization of continuous-time and discrete-time multiple-integrator networks, and propose the adoption of low-gain regulators. We dealt with a fault-tolerant dominat-pole-placement problem for double-integrator networks in [35] and for single-integrator networks in [36]. In this paper, by following the lead of $[30,35,36]$, the solution is attained by means of high-gain regulators. In $[31,32,35]$, the exchange of information among the agents pertained only to the states relative to the highest-order integrals of the control variable. On the contrary, the lower-order integrals were not assumed as available for measure. Here, we remove this assumption, as we did in [33,34].

We show that the problem outlined above can be solved by means of least-order regulators, whose dynamics are spread over the network.

The second problem concerns consensus. We remind the reader that the consensus problems are those where the agents' states are required to asymptotically converge to a unique motion, which may be called agreement law, or else consensus function. Typically, this objective has to be reached by means of a distributed protocol, or, with a terminology more usual in control, by means of a decentralized regulator, which makes the control variable of each agent to depend only on its outputs. These outputs are combinations of the differences of the agents' states, which they share according to a given topology. See, e.g., [13,14].

With respect to standard consensus problems, the one considered here has some particularities. The first one is that the agreement law is given a priori, that is, it is an arbitrarily prespecified linear combination of the agents' initial states (see, e.g., $[15,16]$ ). Note that we do not assume the presence of a leader agent, nor of a model reference (see, e.g., [8,11, 13, 20, 37]). As far as the second specific feature is concerned, we introduce a dominant-pole-placement issue in the problem. With such a requirement, we assign the network dynamics towards what is called the consensus manifold, that is, we assign the shape of the transients towards the agreement law. Note that, on the contrary, only the convergence is usually required, without any particular attention to speed of response and damping (see, e.g., $[7,10]$ ). Finally, the most qualifying aspect of the present problem is that we assume that the communication apparatuses of the agents are subject to faults, and include a fault-tolerance condition. To be more precise, we consider the possibility that one or more couples of agents become unable to exchange the differences of their states, and require that the controlled network still reaches the agreement law with the prescribed dynamics in all these events. Many previous papers deal with what is called switching topology (see, e.g., [9, 15, 17, 37]). However, the invariance with respect to faults of both agreement law and dynamics towards the consensus manifold are usually not required. We here follow the lead of [38], where the agents can have only single- and double-integrator dynamics, and of [39], where the agents have a second-order oscillator dynamics. We propose the use of high-gain regulators even for solving this second problem.

The solution technique for the first problem has its roots in some previous results of ours, concerning what is called regulator problem in the presence of actuator and sensor faults [40-43]. The second problem is solved by exploiting the results found for the first one. 
The paper is organized as follows. We describe the network and the decentralized regulator in the next section. Then, we formally state and solve the fault-tolerant pole-placement problem and the fault-tolerant consensus problem in Section 3 and 4, respectively. Subsequently, we illustrate our design techniques in Section 5 by means of a couple of examples. Finally, we present some concluding remarks in Section 6. The proofs are collected in the Appendix.

Notation. Let $\emptyset$ denote the empty set, and, for any set $\xi$, let $\kappa_{\xi}$ be its cardinality. For any vector $\varphi$, let $[\varphi]_{i}$ denote its $i$-th entry. For any matrix $K$, let $[K]_{i}$ be its $i$-th row and $k_{i, j}$ its $(i, j)$ entry. If $K$ is diagonal, the entries $k_{i, i}$ are denoted by $k_{i}$ for simplicity.

For any matrix $K \in \mathrm{R}^{\sigma \times \sigma}$ and any two sets of integers

$$
\begin{aligned}
& \xi_{1}:=\left\{\xi_{1 i} \mid 1 \leq \xi_{1 i} \leq \sigma \text { and } \xi_{1 i} \neq \xi_{1 j}, \forall i \neq j\right\} \\
& \xi_{2}:=\left\{\xi_{2 i} \mid 1 \leq \xi_{2 i} \leq \sigma \text { and } \xi_{2 i} \neq \xi_{2 j}, \forall i \neq j\right\}
\end{aligned}
$$

let

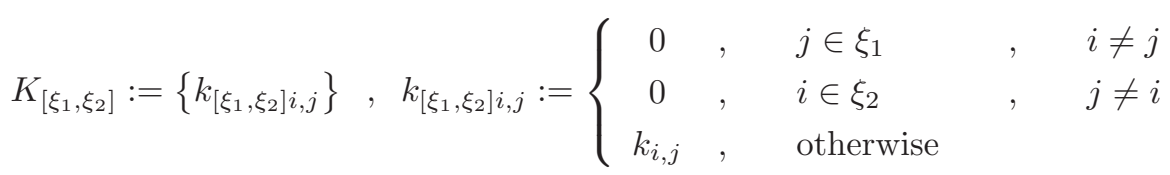

It turns out that $K_{\left[\xi_{1}, \xi_{2}\right]} \in \mathrm{R}^{\sigma \times \sigma}$. Of course, $K_{[\emptyset, \emptyset]}=K$. For any set of integers $\xi$, of the same form as $\xi_{1}$ and $\xi_{2}$ above, let $K_{(\xi)} \in \mathrm{R}^{\left(\sigma-\kappa_{\xi}\right) \times\left(\sigma-\kappa_{\xi}\right)}$ be the matrix obtained from $K$ after removing its rows and columns with indices in the set $\xi$. Analogously, for any vector $\varphi \in \mathrm{R}^{\sigma}$, let $\varphi_{(\xi)} \in \mathrm{R}^{\left(\sigma-\kappa_{\xi}\right)}$ be the vector obtained from $\varphi$ after removing its entries with index in $\xi$. Conventionally, if $\xi=\emptyset$ nothing is removed. In a similar way, for any scalar variable $\gamma$, let us adopt the notations $K_{\left[\xi_{1}, \xi_{2}\right]}(\gamma)$, $K_{(\xi)}(\gamma), \varphi_{(\xi)}(\gamma)$ for a matrix function $K(\gamma)$ and a vector function $\varphi(\gamma)$.

As usual, let $I_{\sigma} \in \mathrm{R}^{\sigma \times \sigma}$ be the $\sigma \times \sigma$ identity matrix and 0 be the zero matrix of any dimensions. Furthermore, let 1 denote the column vector, of any dimension, with all its entries equal to 1 .

A set of negative real and/or pairwise complex conjugate numbers with negative real parts is called Hurwitz.

Finally, let $o(\zeta)$ be any function such that $\lim _{\zeta \rightarrow 0} o(\zeta) / \zeta=0$.

\section{NETWORK AND REGULATOR}

We consider a network of $m>1$ identical agents, whose internal dynamics are constituted by chaines of $n>1$ integrators. As a whole, it is described in the time-domain by

$$
\begin{gathered}
\dot{x}_{i}(t)=x_{i+1}(t), i \in \mathcal{N} \backslash\{n\} \\
\dot{x}_{n}(t)=u(t)
\end{gathered}
$$

where $\mathcal{N}:=\{1,2, \ldots, n\}$. Here, $u(t) \in \mathrm{R}^{m}$ and $x_{j}(t) \in \mathrm{R}^{m}, j \in \mathcal{N}$, are vectors collecting all the control variables and the $j$-th state variables of all the agents, respectively. Of course, $x_{j}(t)$ is the $(n-j+1)$-th integral of $u(t)$. 
We assume that only a partial information on the state of the network is available to the agents. Specifically, each agent has got $p, 1 \leq p \leq n$, scalar outputs. The $j$-th of them, $j \in \mathcal{P}:=$ $\{1,2, \ldots, p\}$, is a linear combination of the entries of $x_{j}(t)$. As a whole, the output of each agent depends on the $p$ highest-order integrals of $u(t)$. Finally, the coefficients of these combinations are the same for all $j \in \mathcal{P}$. Thus, the flow of information throughout the network is formally described by

$$
y_{j}(t)=G x_{j}(t), j \in \mathcal{P}
$$

where $y_{j}(t) \in \mathrm{R}^{m}, j \in \mathcal{P}$, and $G \in \mathrm{R}^{m \times m}$ is real and referred to as the topology matrix. Of course, the input of the $i$-th agent, $i \in \mathcal{M}$, is $[u(t)]_{i}$ and its outputs are $\left[y_{j}(t)\right]_{i}, j \in \mathcal{P}$.

The model (1), (2), where both $n$ and $p$ are arbitrary, has already appeared in [33]. It generalizes those frequently adopted in the literature. See, for instance, [30,35], where $n=2$ and $p=1$, [31,32], where $n$ is arbitrary and $p=1,[12,17]$, where $n$ is arbitrary and $p=n$.

In the complex variable domain, our model can be given the form

$$
Y_{j}(s)=\frac{1}{s^{n-j+1}} G U(s), j \in \mathcal{P}
$$

where $Y_{j}(s)$ and $U(s)$ are the Laplace transforms of $y_{j}(t)$ and $u(t)$.

Our goal is controlling the above network. Specifically, we aim at adopting decentralized linear time-invariant regulators composed of $m$ local elements, the $i$-th of which is applied to the $i$-th agent. Such regulators can be described by

$$
\begin{gathered}
U(s)=\sum_{j \in \mathcal{P}} R_{j}(s) Y_{j}(s) \\
R_{j}(s):=D(s)^{-1} N_{j}(s), j \in \mathcal{P} \\
N_{j}(s):=\operatorname{diag}\left\{n_{j 1}(s), n_{j 2}(s), \ldots, n_{j m}(s)\right\}, j \in \mathcal{P} \\
D(s):=\operatorname{diag}\left\{d_{1}(s), d_{2}(s), \ldots, d_{m}(s)\right\}
\end{gathered}
$$

where $R_{j}(s), j \in \mathcal{P}$, is proper, the $1+p$ polynomials $d_{i}(s), n_{1 i}(s), n_{2 i}(s), \ldots, n_{p i}(s)$ do not have common factors, and the $d_{i}(s) \mathrm{s}$ are monic polynomials of degree $\rho_{i}, i \in \mathcal{M}:=\{1,2, \ldots, m\}$.

Moreover, let

$$
R(s):=\left[\begin{array}{llll}
R_{1}(s) & R_{2}(s) & \cdots & R_{p}(s)
\end{array}\right]
$$

The particular control problems addressed in this paper are stated and solved in the following two sections. The qualifying feature of both of them is that they incorporate tolerance requirements with respect to classes of faults in the communication apparatuses. Such classes of faults will be specified later on. 


\section{FAULT-TOLERANT DOMINANT-POLE-PLACEMENT}

This section is devoted to present a fault-tolerant dominant-pole-placement problem for the previously introduced multiple-integrator network. The problem is stated in the next subsection and solved in the following one.

\subsection{Problem statement}

As already mentioned, our network is subject to faults in the communication apparatuses of one or more agents. Specifically, in this section we assume that the agents with indices in any set $f_{T} \subseteq \mathcal{M}$ become unable to transmit information on their states to other agents, and similarly that the agents with indices in any set $f_{R} \subseteq \mathcal{M}$ become unable to receive information on the states of other agents. Hence, all the operating conditions of the network are described by the pair $\left(f_{T}, f_{R}\right) \subseteq \mathcal{M} \times \mathcal{M}$. Of course, $\left(f_{T}, f_{R}\right)=(\emptyset, \emptyset)$ represents the nominal condition.

Equations (1), (2) and (3) describe the network in nominal condition, whereas we are interested in describing also the situations where the above faults occur. Nominal and faulty conditions can be considered at once by substituting (2) with

$$
y_{j}(t)=G_{\left[f_{T}, f_{R}\right]} x_{j}(t),\left(f_{T}, f_{R}\right) \subseteq \mathcal{M} \times \mathcal{M}, j \in \mathcal{P}
$$

and (3) with

$$
Y_{j}(s)=\frac{1}{s^{n-j+1}} G_{\left[f_{T}, f_{R}\right]} U(s),\left(f_{T}, f_{R}\right) \subseteq \mathcal{M} \times \mathcal{M}, j \in \mathcal{P}
$$

Now, notice that the loop transfer function of the positive feedback system (3), (4a), (4b), obtained by cutting the loop at $U(s)$, is

$$
\widehat{L}(s):=\sum_{j \in \mathcal{P}} R_{j}(s) \frac{1}{s^{n-j+1}} G=D_{L}(s)^{-1} \widehat{N}_{L}(s)
$$

where

$$
\widehat{N}_{L}(s):=\sum_{j \in \mathcal{P}} s^{j-1} N_{j}(s) G, D_{L}(s):=s^{n} D(s)
$$

Then, by letting

$$
Q(s):=D_{L}(s)-\widehat{N}_{L}(s)
$$

for any $\left(f_{T}, f_{R}\right) \subseteq \mathcal{M} \times \mathcal{M}$, the characteristic equation of the positive feedback system (4a), (4b), (7) is

$$
\operatorname{det}\left(Q_{\left[f_{T}, f_{R}\right]}(s)\right)=0
$$

We observe that, for any couple $\left(f_{T}, f_{R}\right) \subseteq \mathcal{M} \times \mathcal{M}$,

$$
\operatorname{det}\left(Q_{\left[f_{T}, f_{R}\right]}(s)\right)=\chi\left(s, f_{T}, f_{R}\right) \prod_{i \in f_{T} \cup f_{R}} \omega_{i}(s)
$$


where

$$
\begin{aligned}
& \chi\left(s, f_{T}, f_{R}\right):=\left\{\begin{array}{cl}
\operatorname{det}\left(Q_{\left(f_{T} \cup f_{R}\right)}(s)\right) & , \quad f_{T} \cup f_{R} \subset \mathcal{M} \\
1 & , \quad f_{T} \cup f_{R}=\mathcal{M}
\end{array}\right. \\
& \omega_{i}(s):=Q_{(\mathcal{M} \backslash\{i\})}(s)=s^{n} d_{i}(s)-\sum_{j \in \mathcal{P}} s^{j-1} n_{j i}(s) g_{i, i}, i \in f_{T} \cup f_{R}
\end{aligned}
$$

Equations (12), (13) can easily be proved by recalling (4c) (4d), (9), (10) and the zero-non-zero pattern of $G_{\left[f_{T}, f_{R}\right]}$. Hence, the characteristic equation (11) of system (4), (7) is equivalent to the set of equations

$$
\begin{gathered}
\chi\left(s, f_{T}, f_{R}\right)=0, f_{T} \cup f_{R} \subset \mathcal{M} \\
\omega_{i}(s)=0, i \in f_{T} \cup f_{R}
\end{gathered}
$$

Equation (14a) is the characteristic equation of the controlled subnetwork composed of the agents not involved in faults. On the other hand, (14b) are the characteristic equations of the single controlled agents whose transmitting and/or receiving apparatuses are faulty.

Then, we are in the right position to state our problem.

\section{Problem 3.1}

Let $m$ Hurwitz sets $\widehat{\mathcal{L}}_{i}:=\left\{\hat{\lambda}_{i j} \mid j \in \mathcal{N} \backslash\{n\}\right\}, i \in \mathcal{M}$, and two positive scalars $\alpha$ and $\beta$ be given. Find a regulator with transfer function $R(s)$ of the form (4b)-(4d), (5) such that, for all $\left(f_{T}, f_{R}\right) \subseteq$ $\mathcal{M} \times \mathcal{M}$, the conditions specified in the following points are satisfied.

1. Equation (14a) possesses $(n-1)\left(m-\kappa_{f_{T} \cup f_{R}}\right)$ roots $\lambda_{i j}$ with

$$
\operatorname{Re}\left\{\lambda_{i j}\right\}<0,\left|\frac{\lambda_{i j}-\hat{\lambda}_{i j}}{\hat{\lambda}_{i j}}\right|<\alpha, i \in \mathcal{M} \backslash\left(f_{T} \cup f_{R}\right), j \in \mathcal{N} \backslash\{n\}
$$

and $m-\kappa_{f_{T} \cup f_{R}}+\sum_{i \in \mathcal{M} \backslash\left(f_{T} \cup f_{R}\right)} \rho_{i}$ roots $\nu_{i r_{i}}$ with

$$
\operatorname{Re}\left\{\nu_{i r_{i}}\right\}<-\beta, r_{i}=1,2, \ldots, \rho_{i}+1, i \in \mathcal{M} \backslash\left(f_{T} \cup f_{R}\right)
$$

2. Each one of (14b) possesses $n-1$ roots $\lambda_{i j}$ with

$$
\operatorname{Re}\left\{\lambda_{i j}\right\}<0,\left|\frac{\lambda_{i j}-\hat{\lambda}_{i j}}{\hat{\lambda}_{i j}}\right|<\alpha, i \in f_{T} \cup f_{R}, j \in \mathcal{N} \backslash\{n\}
$$

and $1+\rho_{i}$ roots $\nu_{i r_{i}}$ with

$$
\operatorname{Re}\left\{\nu_{i r_{i}}\right\}<-\beta, r_{i}=1,2, \ldots, \rho_{i}+1, i \in f_{T} \cup f_{R}
$$

This problem can be interpreted as the formal statement of a dominant-pole-placement issue raised in both nominal and faulty conditions. The elements $\hat{\lambda}_{i j}$ of the $\widehat{\mathcal{L}}_{i}$ s are the desired dominant poles, whereas $\alpha$ and $\beta$ are design parameters. More precisely, Problem 3.1 requires that the 
controlled network possesses $(n-1) m$ eigenvalues $\lambda_{i j}$ located close to the $\hat{\lambda}_{i j}$ s. The parameter $\alpha$ specifies how close they have to be (see (15), (17)). Of course, it is reasonable that $\alpha$ is small. Furthermore, the location of the $m+\sum_{i \in \mathcal{M}} \rho_{i}$ remaining eigenvalues is constrained by (16) and (18). If $\beta \gg \max _{i \in \mathcal{M}, j \in \mathcal{N} \backslash\{n\}}\left|\operatorname{Re}\left(\hat{\lambda}_{i j}\right)\right|$, then the $\lambda_{i j}$ s can legitimately be interpreted as the dominant poles of the controlled network. Since the $\hat{\lambda}_{i j}$ s are arbitrary, the initial condition response of the overall control system can be made as fast as desired. We stress that the above must be true not only in nominal, but also in all possible faulty conditions.

\subsection{Problem solution}

An obvious necessary condition for Problem 3.1 solvability is that matrix $G$ is nonsingular, since, otherwise, the network is not observable in nominal condition. Furthermore, the results in $[44,45]$ guarantee that appropriate pole-placement problems can be solved for any fault $\left(f_{T}, f_{R}\right) \subseteq \mathcal{M} \times \mathcal{M}$ by means of decentralized regulators tuned to $\left(f_{T}, f_{R}\right)$ if and only if $G_{\left[f_{T}, f_{R}\right]}$ is nonsingular. Indeed, this condition means that the faulty network has no fixed modes (at $s=0$ ). However, the regulator transfer function (4b)-(4d), (5) is independent of the network operating condition. Put in a different way, Problem 3.1 calls for the existence of a single decentralized regulator able to solve at once all the dominant-pole-placement problems corresponding to all the possible (nominal and faulty) network operating conditions. Hence, without loss of generality, we assume

$$
\operatorname{det}\left(G_{\left[f_{T}, f_{R}\right]}\right) \neq 0,\left(f_{T}, f_{R}\right) \subseteq \mathcal{M} \times \mathcal{M}
$$

and expect a solvability condition tighter than (19).

In this subsection, our line of reasoning, aimed at solving Problem 3.1, is the following. First, we state the new Problem 3.2 and prove its equivalence with Problem 3.1 (Lemma 3.1). Then, we study the solvability of Problem 3.2 (Lemmas 3.2-3.4). Finally, we actually give the necessary and sufficient condition for the solvability of Problem 3.1 and supply explicit formulas for the regulator (Theorem 3.1).

As a first step, for any given $f \subset \mathcal{M}$, we define the equation

$$
\operatorname{det}\left(Q_{(f)}(s)\right)=0
$$

Then, we state the following problem.

Problem 3.2

Let $m$ Hurwitz sets $\widehat{\mathcal{L}}_{i}:=\left\{\hat{\lambda}_{i j} \mid j \in \mathcal{N} \backslash\{n\}\right\}, i \in \mathcal{M}$, and two positive scalars $\alpha$ and $\beta$ be given. Find a regulator with transfer function $R(s)$ of the form (4b)-(4d), (5) such that, for all $f \subset \mathcal{M}$, (20) possesses $(n-1)\left(m-\kappa_{f}\right)$ roots $\lambda_{i j}$ with

$$
\operatorname{Re}\left\{\lambda_{i j}\right\}<0,\left|\frac{\lambda_{i j}-\hat{\lambda}_{i j}}{\hat{\lambda}_{i j}}\right|<\alpha, i \in \mathcal{M} \backslash f, j \in \mathcal{N} \backslash\{n\}
$$

and $m-\kappa_{f}+\sum_{i \in \mathcal{M} \backslash f} \rho_{i}$ roots $\nu_{i r_{i}}$ with

$$
\operatorname{Re}\left\{\nu_{i r_{i}}\right\}<-\beta, r_{i}=1,2, \ldots, \rho_{i}+1, i \in \mathcal{M} \backslash f
$$


The relationship between Problem 3.1 and Problem 3.2 is enlightened by the following result.

\section{Lemma 3.1}

Problem 3.1 admits a solution if and only if Problem 3.2 admits a solution.

Furthermore, a regulator $R(s)$ of the form (4b)-(4d), (5) solves Problem 3.1 if and only if it solves Problem 3.2.

The rationale behind Problem 3.2 and Lemma 3.1 is fairly easy to understand. We have already observed that, when a fault $\left(f_{T}, f_{R}\right)$ occurs, the characteristic equation of the network breaks down into the equations (14). Then, even the dominant-pole-placement problem breaks down into subproblems relative to the single factors of the overall characteristic polynomial. Moreover, considering all the $f \subset \mathcal{M}$ in Problem 3.2 means that all the $\left(f_{T}, f_{R}\right) \subseteq \mathcal{M} \times \mathcal{M}$ in Problem 3.1 are considered. The details are in the proof.

Now, we state a necessary condition for the solvability of Problem 3.2. It makes reference to the matrix

$$
\widetilde{G}:=\operatorname{diag}\left\{\frac{g_{1,1}}{\left|g_{1,1}\right|}, \frac{g_{2,2}}{\left|g_{2,2}\right|}, \ldots, \frac{g_{m, m}}{\left|g_{m, m}\right|}\right\}
$$

which is well defined, since (19) implies $g_{i, i} \neq 0, i \in \mathcal{M}$.

\section{Lemma 3.2}

Problem 3.2 admits a solution only if

$$
\operatorname{det}\left(G_{(f)}\right) \operatorname{det}\left(\widetilde{G}_{(f)}\right)>0, \forall f \subset \mathcal{M}
$$

A moment reflection allows us to realize that condition (24) includes (19), for all $\left(f_{T}, f_{R}\right) \subseteq$ $\mathcal{M} \times \mathcal{M}$, and is actually tighter than it, as expected.

Let us move now towards a sufficient solvability condition for Problem 3.2, by first defining the scalars $\hat{e}_{i j}$ through the identity

$$
\sum_{j \in \mathcal{N}} \hat{e}_{i j} s^{j-1} \equiv \prod_{j \in \mathcal{N} \backslash\{n\}}\left(s-\hat{\lambda}_{i j}\right), i \in \mathcal{M}
$$

Furthermore, let

$$
\begin{aligned}
& \widehat{E}_{j}:=\operatorname{diag}\left\{\hat{e}_{1 j}, \hat{e}_{2 j}, \ldots, \hat{e}_{m j}\right\} \in \mathrm{R}^{m \times m}, j \in \mathcal{N} \\
& \widehat{E}_{S}:=\left[\begin{array}{lllll}
\widehat{E}_{1} & \widehat{E}_{2} & \cdots & \widehat{E}_{p-1} & 0
\end{array}\right] \in \mathrm{R}^{m \times p m}
\end{aligned}
$$

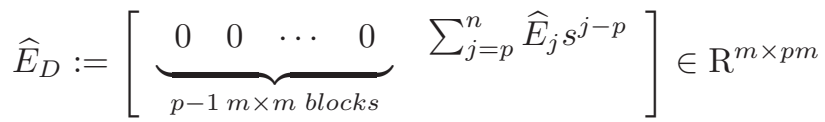

Note that $\hat{e}_{i n}=1, i \in \mathcal{M}$, so that $\widehat{E}_{n}=I_{m}$.

Then, for any $\mu \neq 0, \varepsilon \neq 0$ and $\widehat{V} \in \mathrm{R}^{m \times m}$, consider the regulator

$$
R(s)=\mu \widehat{V}\left\{\widehat{E}_{S}+\frac{1}{(1+\varepsilon s)^{n-p}} \widehat{E}_{D}(s)\right\}
$$


It is easy to check that such regulator is characterized by $\rho_{i}=n-p, i \in \mathcal{M}$, and can be given the realization

$$
\begin{array}{r}
\varepsilon \dot{\hat{z}}_{i}(t)=-\hat{z}_{i}(t)+\hat{z}_{i+1}(t)+\widehat{H}_{i} \widehat{V} y_{p}(t), i=1,2, \ldots, n-p-1 \\
\varepsilon \dot{\hat{z}}_{n-p}(t)=-\hat{z}_{n-p}(t)+\widehat{H}_{n-p} \widehat{V} y_{p}(t) \\
u(t)=\mu\left\{\sum_{j \in \mathcal{P} \backslash\{p\}} \widehat{E}_{j} \widehat{V} y_{j}(t)+\varepsilon^{-(n-p)} \widehat{V} y_{p}(t)-\varepsilon^{-(n-p)} \hat{z}_{1}(t)\right\}
\end{array}
$$

where $\hat{z}_{i}(t) \in \mathrm{R}^{m}, i=1,2, \ldots, n-p$, whereas the coefficients $\widehat{H}_{i} \in \mathrm{R}^{m \times m}, i=1,2, \ldots, n-p$, are uniquely defined through the identity

$$
(1+\varepsilon s)^{n-p}-\sum_{i=1}^{n-p} \widehat{H}_{i}(1+\varepsilon s)^{n-p-i} \equiv \varepsilon^{n-p} \sum_{j=p}^{n} \widehat{E}_{j} s^{j-p}
$$

Hence, for any $f \subset \mathcal{M}$, (20) is the characteristic equation of the controlled subnetwork described in the time-domain by

$$
\begin{gathered}
\dot{x}_{i(f)}(t)=x_{i+1(f)}(t), i \in \mathcal{N} \backslash\{n\} \\
\dot{x}_{n(f)}(t)=\mu\left\{\sum_{j \in \mathcal{P} \backslash\{p\}} \widehat{E}_{i(f)} \widehat{V}_{(f)} G_{(f)} x_{j(f)}(t)+\varepsilon^{-(n-p)} \widehat{V}_{(f)} G_{(f)} x_{p(f)}(t)-\varepsilon^{-(n-p)} \hat{z}_{1(f)}(t)\right\} \\
\varepsilon \dot{\hat{z}}_{i(f)}(t)=-\hat{z}_{i(f)}(t)+\hat{z}_{i+1(f)}(t)+\widehat{H}_{i(f)} \widehat{V}_{(f)} G_{(f)} x_{p(f)}(t), i=1,2, \ldots, n-p-1 \\
\varepsilon \dot{\hat{z}}_{n-p(f)}(t)=-\hat{z}_{n-p(f)}(t)+\widehat{H}_{n-p(f)} \widehat{V}_{(f)} G_{(f)} x_{p(f)}(t)
\end{gathered}
$$

We are now ready to state a first sufficient condition for the solvability of Problem 3.2.

\section{Lemma 3.3}

If there exists a diagonal matrix $\widehat{V}$ such that, for all $f \subset \mathcal{M}$, the matrices $\widehat{V}_{(f)} G_{(f)}$ are Hurwitz, then Problem 3.2 admits a solution.

Furthermore, there exist $\hat{\mu}>0$ and $\hat{\varepsilon}(\cdot)>0$ such that, for all $\mu>\hat{\mu}$ and $\varepsilon \in(0, \hat{\varepsilon}(\mu))$, the regulator (25)-(29) solves Problem 3.2.

Thus, whenever matrix $\widehat{V}$ there exists, the regulator (25)-(29) with $\mu$ sufficiently large and $\varepsilon$ sufficiently small, is able to place the eigenvalues of systems (30) so that they satisfy conditionts (21), (22) of Problem 3.2 for all $f \subset \mathcal{M}$.

A first remark on Lemma 3.3 concerns the rationale behind it, which is the following. For any $f \subset \mathcal{M}$, (20) is the characteristic equation of the positive feedback system with loop transfer function $\widehat{L}_{(f)}(s)$, where $\widehat{L}(s)$ is given by (8). In view of (5), (8), (25)-(27), it turns out that

$$
\widehat{L}_{(f)}(s)=\frac{\mu}{s^{n}} \widehat{V}_{(f)}\left(\sum_{j \in \mathcal{P} \backslash\{p\}} \widehat{E}_{j(f)} s^{j-1}+\frac{1}{(1+\varepsilon s)^{n-p}} \sum_{j=p}^{n} \widehat{E}_{j(f)} s^{j-1}\right) G_{(f)}
$$


If we first set $\varepsilon=0$ in (27), the regulator turns out to be nondynamic and to contain multiple derivative actions. In this situation, when $\mu \rightarrow+\infty$, the closed-loop system has $(n-1)\left(m-\kappa_{f}\right)$ eigenvalues satisfying (21). This is due to the fact that the regulator introduces $(n-1)\left(m-\kappa_{f}\right)$ transmission zeros in the locations where the dominant poles have to be placed, as it is apparent from (31). Furthermore, the remaining $m-\kappa_{f}$ eigenvalues satisfy (22). Indeed, (27) shows that they tend to the eigenvalues of $\mu \widehat{V}_{(f)} G_{(f)}$, which is Hurwitz. Then, by letting $\varepsilon$ be positive and sufficiently small, the previously mentioned eigenvalues still satisfy their respective conditions, the regulator becomes proper, and $(n-p)\left(m-\kappa_{f}\right)$ new eigenvalues appear, which satisfy (22). The details are in the proof.

Furthermore, the regulator of Lemma 3.3 has got a number of interesting features. First of all, it is indeed decentralized, since the matrices $\widehat{V}$ and $\widehat{E}_{j}, j \in \mathcal{N}$, are actually diagonal. Secondly, it is high-gain, in the sense that its d.c. gain goes to infinity together with $\mu$. However, what is most important is that its order $(n-p) m$ is the least possible one. In fact, consider the case $f=\mathcal{M} \backslash\{i\}$. Then, (20) becomes $\omega_{i}(s)=0$, that is,

$$
s^{n} d_{i}(s)-\sum_{j \in \mathcal{P}} s^{j-1} n_{j i}(s) g_{i, i}=0
$$

A necessary condition for this equation to have all the roots lying in the open left-half of the complex plane is that the coefficient of $s^{n-1}$ in $\omega_{i}(s)$ is different from zero. This implies that the degree of some $n_{j i}(s)$ is at least $n-p$, which, at its own turn, implies that the degree of $d_{i}(s)$ is not less than $n-p$, since the regulator (4b)-(4d) is assumed as proper. Hence, the order of the regulator (4b)-(4d) cannot be less than $(n-p) m$, which is the order of the regulator of Lemma 3.3.

Moreover, observe that all the dynamics are concentrated in the regulator transfer function between $y_{p}(t)$ and $u(t)$, whereas the transfer functions between $y_{i}(t), i \in \mathcal{P} \backslash\{p\}$, and $u(t)$ are nondynamic. Coherently, when $p=n$ (all network state variables are available for measure), it turns out

$$
R(s)=\mu \widehat{V}\left[\begin{array}{llll}
\widehat{E}_{1} & \widehat{E}_{2} & \cdots & \widehat{E}_{n}
\end{array}\right]
$$

and, when $p=1$ (only $x_{1}(t)$ is available for measure), it turns out

$$
R(s)=\frac{\mu}{(1+\varepsilon s)^{n-1}} \widehat{V} \sum_{j \in \mathcal{N}} \widehat{E}_{j} s^{j-1}
$$

Lemma 3.3 gives a sufficient solvability condition not expressed in terms of Problem 3.2 data and, as such, cannot be said completely satisfactory. On the contrary, it raises the subproblem of the existence of $\widehat{V}$. Fortunately enough, we can give a complete solution to this subproblem in the following lemma, which is based on a general algebraic result of ours [46].

\section{Lemma 3.4}

A diagonal matrix $\widehat{V}$ there exists such that, for all $f \subset \mathcal{M}$, the matrices $\widehat{V}_{(f)} G_{(f)}$ are Hurwitz if and only if (24) holds.

Furthermore, if (24) holds, then there exists $\hat{\tau}>0$ such that, for all $f \subset \mathcal{M}$ and for all $\tau \in(0, \hat{\tau})$, the matrices $-\widetilde{G}_{(f)} \mathrm{T}_{(f)} G_{(f)}$, where 


$$
\mathrm{T}:=\operatorname{diag}\left\{\tau, \tau^{2}, \ldots, \tau^{m}\right\}
$$

have distinct negative real eigenvalues.

Hence, it is clear that, for any $\tau \in(0, \hat{\tau})$, the matrix

$$
\widehat{V}:=-\widetilde{G} \mathrm{~T}(\tau)
$$

has the property required in Lemma 3.3.

As a consequence, the necessary condition (24) is also sufficient for the existence of a solution to Problem 3.2. Thus, the combination of Lemmas 3.1-3.4 supplies the proof of the main result of this section, stated in the following theorem.

\section{Theorem 3.1}

Problem 3.1 admits a solution if and only if (24) holds.

Furthermore, there exist $\hat{\tau}>0, \hat{\mu}(\cdot)>0$ and $\hat{\varepsilon}(\cdot)>0$ such that, for all $\tau \in(0, \hat{\tau})$, for all $\mu>\hat{\mu}(\tau)$ and for all $\varepsilon \in(0, \hat{\varepsilon}(\mu))$, the regulator (23), (25)-(29), (32), (33) solves Problem 3.1.

It is worth noticing that the necessary and sufficient solvability condition (24) depends only on the topology matrix $G$. On the contrary, it is independent of $n$ and $p$. Condition (24) has already appeared in the literature, though in the equivalent form $\operatorname{det}\left(G_{(f)}\right) \prod_{i \in \mathcal{M} \backslash f} g_{i, i}>0, f \in \mathcal{M}$. For instance, it was expedient in [35] and [36] to solve Problem 3.1 for the particular cases $n=2, p=1$ and $n=p=1$, respectively. Quite unexpectedly, the same condition was found to be necessary and sufficient to solve the less demanding fault-tolerant stabilization problem dealt with in [31] for $n$ arbitrary, $p=1$, and in [33] for $n$ and $p$ arbitrary. Finally, it coincides as well with the solvability condition for the fault-tolerant stabilization problem stated for discrete-time chaines of integrators in [32], where $n$ is arbitrary and $p=1$, and in [34], where $n$ and $p$ are arbitrary. On the other hand, the regulator (23), (25)-(29), (32), (33) does depend on $n$ and $p$, and, as already noticed, its order is the least possible one for any $n$ and $p$.

One may wonder whether condition (24) can be given any interpretation in terms of the existence of some special paths (e.g., trees) in the graph of the network, which is the graph having a node for each agent and an edge from node $i$ to node $j$ if the output of agent $j$ depends on the state of agent $i$. Quite differently from what happens in consensus problems (see, e.g., the next section of this paper), the answer is negative. This claim is immediately proved by considering the limit case of a diagonal $G$, with any nonzero diagonal entries, where condition (24) always holds. However, the fulfilment of condition (24) depends in general on the specific values of the entries of $G$, not only on its zero-non-zero pattern. For instance, when $m=2$, condition (24) takes on the form $g_{1,1} g_{2,2}\left(g_{1,1} g_{2,2}-g_{1,2} g_{2,1}\right)>0$.

We also point out that the regulator (23), (25)-(29), (32), (33) solving Problem 3.1 can be designed according to the following three-step procedure: (i) find a sufficiently small positive $\tau$ such that the matrices $-\widetilde{G}_{(f)} \mathrm{T}_{(f)} G_{(f)}, f \subset \mathcal{M}$, are Hurwitz; (ii) set $\varepsilon=0$ and find a sufficiently large $\mu$ such that (15)-(18) are satisfied; (iii) find a sufficiently small positive $\varepsilon$ such that (15)-(18) still hold. The possibility of successfully performing step (i) is guaranteed by Lemma 3.4. As far as steps (ii) and (iii) are concerned, their feasibility is guaranteed by Lemma 3.3, as a consequence of its already illustrated rationale. 
Finally, it is worth noticing that our solution to Problem 3.1 supplies the controlled network with a nice robustness property. Specifically, let

$$
\begin{gathered}
\Delta_{l}:=\operatorname{diag}\left\{\delta_{l 1}, \delta_{l 2}, \ldots, \delta_{l m}\right\} \\
\Delta_{r}:=\operatorname{diag}\left\{\delta_{r 1}, \delta_{r 2}, \ldots, \delta_{r m}\right\} \\
0<\delta_{l i} \leq \hat{\delta}_{l i}, \hat{\delta}_{l i} \geq 1,0<\delta_{r i} \leq \hat{\delta}_{r i}, \hat{\delta}_{r i} \geq 1, i \in \mathcal{M}
\end{gathered}
$$

and consider a matrix $G$ which takes on the form

$$
G=\Delta_{l} G_{n} \Delta_{r}
$$

where $G_{n}$ can be thought of as the known nominal topology matrix.

Then, we consider Problem 3.1, where the conditions in points 1 and 2 have to be satisfied for $G$ as in (35) and all $\Delta_{l}$ and $\Delta_{r}$ specified by (34).

This modified problem seems interesting, since matrices $\Delta_{l}$ and $\Delta_{r}$ allow us to take into account possible uncertainties on the gains of the communication apparatuses. For instance, assuming that $\hat{\delta}_{l i}=1\left(\hat{\delta}_{r i}=1\right)$ means that the gain of the receiving (transmitting) apparatus of the $i$-th agent may undergo any unknown reduction $\delta_{l i}\left(\delta_{r i}\right)$ with respect to the gain specified by $G_{n}$ (see (2)).

Now, we observe that, in view of (35), (34),

$$
\operatorname{det}\left(G_{(f)}\right) \operatorname{det}\left(\widetilde{G}_{(f)}\right)=\operatorname{det}\left(\Delta_{l(f)}\right) \operatorname{det}\left(G_{n(f)}\right) \operatorname{det}\left(\Delta_{r(f)}\right) \operatorname{det}\left(\widetilde{G}_{n(f)}\right)
$$

where

$$
\widetilde{G}_{n}:=\operatorname{diag}\left\{\frac{g_{n 1,1}}{\left|g_{n 1,1}\right|}, \frac{g_{n 2,2}}{\left|g_{n 2,2}\right|}, \ldots, \frac{g_{n m, m}}{\left|g_{n m, m}\right|}\right\}
$$

Since $\operatorname{det}\left(\Delta_{l(f)}\right)>0, \operatorname{det}\left(\Delta_{r(f)}\right)>0$, we concludes that the necessary and sufficient solvability condition (24) becomes

$$
\operatorname{det}\left(G_{n(f)}\right) \operatorname{det}\left(\widetilde{G}_{n(f)}\right)>0, f \subset \mathcal{M}
$$

which is independent of $\Delta_{l}$ and $\Delta_{r}$. Consequently, this new problem is solvable if and only if Problem 3.1 is solvable with $G=G_{n}$. Moreover, the regulator (23), (25)-(29), (32), (33), with $G=G_{n}$, solves also our new problem. Of course, tuning the parameters $\tau, \mu$ and $\varepsilon$ is now somehow more cumbersome.

\section{FAULT-TOLERANT CONSENSUS}

Stabilization problems, as the one dealt with in the preceding section, can by no means be solved when matrix $G$ is singular. On the contrary, a different and meaningful class of problems can be faced in this case, provided that $G$ is endowed with some special features. Here, reference is made to what are called consensus problems, which consist in finding regulators such that, for each $j \in \mathcal{N}$, all the components of $x_{j}(t)$ asymptoticallly converge to the same function of time. 
This section is devoted to present a particular fault-tolerant consensus problem for the previously introduced multiple-integrator network. The problem is stated in the next subsection and solved in the following one.

\subsection{Problem statement}

We consider networks (1), (2) and (3) where, for any $j \in \mathcal{P}$, the output $\left[y_{j}(t)\right]_{k}$ of agent $k$ is a nonnegative linear combination of the differences between $\left[x_{j}(t)\right]_{k}$ and $\left[x_{j}(t)\right]_{i}, i \neq k$. Moreover, we assume that the coefficients of the above combinations are independent of $j$ and symmetric. More precisely, let

$$
\begin{gathered}
{\left[y_{j}(t)\right]_{k}=\sum_{i \in \mathcal{M} \backslash\{k\}} l_{k i}\left(\left[x_{j}(t)\right]_{k}-\left[x_{j}(t)\right]_{i}\right), k \in \mathcal{M}, j \in \mathcal{P}} \\
l_{k i}=l_{i k} \geq 0,(k, i) \in \mathcal{M} \times \mathcal{M}, k \neq i
\end{gathered}
$$

The meaning of the symmetry condition (36b) is twofold. First, the communication channel between any two agents is bidirectional, if it there exists. Second, the weight $l_{k i}$ of the difference $\left[x_{j}(t)\right]_{k}-$ $\left[x_{j}(t)\right]_{i}$ in the output $\left[y_{j}(t)\right]_{k}$ is the same as the weight $l_{i k}$ of the difference $\left[x_{j}(t)\right]_{i}-\left[x_{j}(t)\right]_{k}$ in the output $\left[y_{j}(t)\right]_{i}$.

Thus, the topology matrix $G$ of (2) and (3) takes on the form

$$
G:=\left\{g_{k, i}\right\}, g_{k, i}:=\left\{\begin{array}{cl}
-l_{k i} & , \quad k \neq i \\
\sum_{i \in \mathcal{M} \backslash\{k\}} l_{k i} & , \quad k=i
\end{array},(k, i) \in \mathcal{M} \times \mathcal{M}\right.
$$

and, in view of (36), satisfies the following assumption.

\section{Assumption 4.1}

The topology matrix $G$ is such that

$$
\begin{gathered}
g_{k, i} \leq 0,(k, i) \in \mathcal{M} \times \mathcal{M}, k \neq i \\
\sum_{i \in \mathcal{M}} g_{k, i}=0, k \in \mathcal{M} \\
G^{\prime}=G
\end{gathered}
$$

Equations (38a), (38b) define what is usually called a Laplacian matrix. Note that $G$ is singular and $G \mathbf{1}=0$, as a consequence of (38b). Of course, $\mathbf{1}^{\prime} G=0$ too, because of (38c).

As already announced, we assume that faults may occur in the communication apparatuses of the agents. Differently from what has been done in Section 3, we here suppose that one or more couples $(k, i), k \neq i$, of agents may become unable to share the information concerning their states, that is, $l_{k i}=l_{i k}$ may vanish. The consequence of such a fault on the topology matrix is that the entries $(k, i)$ and $(i, k)$ have to be set to zero. Furthermore, the $k$-th and $i$-th diagonal entries have to be recalculated. From a technical point of view, a fault is defined as any couple $\theta \subset \Theta$, where 


$$
\Theta:=\{(k, i) \mid(k, i) \in \mathcal{M} \times \mathcal{M}, i>k\}
$$

Then, when a fault $\theta$ occurs, matrix $G$ has to be substituted with

$$
g_{\langle\theta\rangle k, i}:=\left\{\begin{array}{ccc}
G_{\langle\theta\rangle}:=G_{\langle\theta\rangle}^{\prime}:=\left\{g_{\langle\theta\rangle k, i}\right\} & \\
-\left(\sum_{j \in \mathcal{M},(k, j) \notin \theta,(j, k) \notin \theta} g_{k, j}\right) & , \quad k=i \\
0 & , \quad(k, i) \in \theta \quad, k \in \mathcal{M}, k \leq i \leq m \\
g_{k, i} & , \quad(k, i) \in \Theta \backslash \theta
\end{array}\right.
$$

We observe that $G_{\langle\theta\rangle}$ is Laplacian and symmetric whenever $G$ satisfies Assumption 4.1. Moreover, $G_{\langle\emptyset\rangle}=G$. As a consequence, we can deal with nominal and faulty conditions at once by substituting $G_{\langle\theta\rangle}$ to $G$ whenever it occurs. Specifically, we substitute (2) with

$$
y_{j}(t)=G_{\langle\theta\rangle} x_{j}(t), \theta \subset \Theta, j \in \mathcal{P}
$$

and (3) with

$$
Y_{j}(s)=\frac{1}{s^{n-j+1}} G_{\langle\theta\rangle} U(s), \theta \subset \Theta, j \in \mathcal{P}
$$

The loop transfer function of the positive feedback system (4a), (4b), (40), obtained by cutting the loop at $U(s)$, is

$$
\bar{L}(s, \theta):=\sum_{j \in \mathcal{P}} R_{j}(s) \frac{1}{s^{n-j+1}} G_{\langle\theta\rangle}=D_{L}(s)^{-1} \bar{N}_{L}(s, \theta)
$$

where

$$
\bar{N}_{L}(s, \theta):=\sum_{j \in \mathcal{P}} s^{j-1} N_{j}(s) G_{\langle\theta\rangle}
$$

and $D_{L}(s)$ is given by (9).

Then, for any $\theta \subset \Theta$, the characteristic equation of the positive feedback system (4a), (4b), (40) is

$$
\operatorname{det}\left(D_{L}(s)-\bar{N}_{L}(s, \theta)\right)=0
$$

We remark that, whichever the regulator may be, this equation has at least $n$ roots equal to 0 . Indeed, all the eigenvalues of the network (1), (39) are zero, and straightforward calculations show that the dimension of its unobservability subspace is at least $n$.

Now, we are ready to introduce our fault-tolerant consensus problem with arbitrary assignment of both the agreement law and the dominant dynamics. In its statement, we make reference to the vector

$$
c:=\left[\begin{array}{llll}
c_{1} & c_{2} & \cdots & c_{m}
\end{array}\right]^{\prime}, c_{i}>0, i \in \mathcal{M}, c^{\prime} \mathbf{1}=1
$$




\section{Problem 4.1}

Let $G$ satisfy Assumption 4.1. Furtermore, let an Hurwitz set $\overline{\mathcal{L}}:=\left\{\bar{\lambda}_{j} \mid j \in \mathcal{N} \backslash\{n\}\right\}$, and two positive scalars $\alpha$ and $\beta$ be given. Finally, let $\bar{\Theta}$ be a given proper subset of the power set of $\Theta$, with $\emptyset \in \bar{\Theta}$.

Find a regulator with transfer function $R(s)$ of the form (4b)-(4b), (5) and set its initial state so that, for all $\theta \in \bar{\Theta}$, the conditions specified in the following points are satisfied.

1. For any given $c$ as in (42) and for all $x_{i}(0), i \in \mathcal{N}$,

$$
\lim _{t \rightarrow+\infty}\left(x_{i}(t)-\mathbf{1} c^{\prime} \sum_{k=i}^{n} \frac{t^{k-i}}{(k-i) !} x_{k}(0)\right)=0, i \in \mathcal{N}
$$

2. Equation (41) possesses $(n-1)(m-1)$ roots $\lambda_{i j}$ with

$$
\operatorname{Re}\left\{\lambda_{i j}\right\}<0,\left|\frac{\lambda_{i j}-\bar{\lambda}_{i j}}{\bar{\lambda}_{i j}}\right|<\alpha, i \in \mathcal{M} \backslash\{m\}, j \in \mathcal{N} \backslash\{n\}
$$

and $m-1+\sum_{i \in \mathcal{M}} \rho_{i}$ roots $\nu_{i}$ with

$$
\operatorname{Re}\left\{\nu_{i}\right\}<-\beta, i=1,2, \ldots, m-1+\sum_{i \in \mathcal{M}} \rho_{i}
$$

Point 1 of Problem 4.1 calls for the controlled network to reach a consensus and specifies the desired agreement law. For any $i \in \mathcal{N}$, such agreement law is a polynomial function of $t$ whose coefficients are linear combinations of the network initial states $x_{k}(0), i \leq k \leq n$. The weights of the entries of the $x_{k}(0) \mathrm{s}$ in these combinations are the entries of $c$ divided by $(k-i)$ !. One may wonder whether the agreement law (43) is the only achievable one. The answer is negative. Indeed, it is easily shown that, since $G$ is singular, the uncontrolled network has got (at least) $n$ fixed modes [45] at $s=0$. Furthermore, the controlled system contains (at least) a chain of $n$ unobservable integrators. Hence, the agreement law must be a polynomial function of $t$, and, in general, its coefficients depend on the initial state of both the network and the regulator (see, e.g., [16]). However, the case where the agreement law does not depend on the regulator initial state is of obvious particular interest. Simple computations show that imposing such property implies that the agreement law must have the form in (43). Finally, it is plainly reasonable that the coefficients of $t^{k-i} /(k-i)$ ! in (43) are positive convex combinations of all the entries of $x_{k}(0)$. This is precisely what the definition of $c$ in (42) states.

Point 2 concerns only $n(m-1)+\sum_{i \in \mathcal{M}} \rho_{i}$ eigenvalues out of $n m+\sum_{i \in \mathcal{M}} \rho_{i}$ total ones. As a matter of fact, nothing could be required on the remaining $n$ eigenvalues, since they are anyhow located at $s=0$. Moreover, such condition is entirely equivalent to points 1,2 of Problem 3.1, so that the comment reported just after Problem 3.1 applies also to Problem 4.1. Hence, (44), (45) can legitimately be interpreted as stating a dominant-pole-placement issue for the dynamics towards the consensus manifold. However, we note that in Problem 3.1 the desired locations of the dominant poles are independent one from each other. Indeed, they are the elements of $\widehat{\mathcal{L}}_{i}, i \in \mathcal{M}$. On the other hand, in Problem 4.1 the desired locations of the dominant poles is somehow constrained, since they are the elements of $\overline{\mathcal{L}}$ repeated $m$ times. 
We stress that all the above must be true not only in nominal condition, but also in all the possible considered faulty conditions, as specified by the set $\bar{\Theta}$.

\subsection{Problem solution}

In order to introduce our necessary and sufficient solvability condition for Problem 4.1, we first define the scalars $\bar{e}_{j}$ through the identity

$$
\sum_{j \in \mathcal{N}} \bar{e}_{j} s^{j-1} \equiv \prod_{j \in \mathcal{N} \backslash\{n\}}\left(s-\bar{\lambda}_{j}\right)
$$

Furthermore, let

$$
\begin{gathered}
\bar{E}_{j}:=\bar{e}_{j} I_{m} \in \mathrm{R}^{m \times m}, j \in \mathcal{N} \\
\bar{E}_{S}:=\left[\begin{array}{lllll}
\bar{E}_{1} & \bar{E}_{2} & \cdots & \bar{E}_{p-1} & 0
\end{array}\right] \in \mathrm{R}^{m \times p m} \\
\bar{E}_{D}(s):=\left[\begin{array}{ccccc}
\underbrace{0}_{p-1 m \times m \text { blocks }} & 0 & \cdots & 0 & \sum_{j=p}^{n} \bar{E}_{j} s^{j-p}
\end{array}\right] \in \mathrm{R}^{m \times p m}
\end{gathered}
$$

Note that $\bar{e}_{i n}=1, i \in \mathcal{M}$, so that $\bar{E}_{n}=I_{m}$.

Then, for any $\mu \neq 0$ and $\varepsilon \neq 0$, consider the regulator

$$
\begin{gathered}
R(s)=\mu \bar{V}\left\{\bar{E}_{S}+\frac{1}{(1+\varepsilon s)^{n-p}} \bar{E}_{D}(s)\right\} \\
\bar{V}:=-\operatorname{diag}\left\{c_{1}^{-1}, c_{2}^{-1}, \ldots, c_{m}^{-1}\right\}
\end{gathered}
$$

It is easy to check that such regulator is characterized by $\rho_{i}=n-p, i \in \mathcal{M}$, and can be given the realization

$$
\begin{array}{r}
\varepsilon \dot{\bar{z}}_{i}(t)=-\bar{z}_{i}(t)+\bar{z}_{i+1}(t)+\bar{h}_{i} \bar{V} y_{p}(t), i=1,2, \ldots, n-p-1 \\
\varepsilon \dot{\bar{z}}_{n-p}(t)=-\bar{z}_{n-p}(t)+\bar{h}_{n-p} \bar{V} y_{p}(t) \\
u(t)=\mu\left\{\sum_{j \in \mathcal{P} \backslash\{p\}} \bar{e}_{j} \bar{V} y_{j}(t)+\varepsilon^{-(n-p)} \bar{V} y_{p}(t)-\varepsilon^{-(n-p)} \bar{z}_{1}(t)\right\}
\end{array}
$$

where $\bar{z}_{i}(t) \in \mathrm{R}^{m}, i=1,2, \ldots, n-p$, whereas the scalars $\bar{h}_{i}, i=1,2, \ldots, n-p$, are uniquely defined through the identity

$$
(1+\varepsilon s)^{n-p}-\sum_{i=1}^{n-p} \bar{h}_{i}(1+\varepsilon s)^{n-p-1} \equiv \varepsilon^{n-p} \sum_{j=p}^{n} \bar{e}_{j} s^{j-p}
$$

Then, the solution of our fault-tolerant consensus problem is given in the following theorem. 


\section{Theorem 4.1}

Problem 4.1 admits a solution if and only if the set $\bar{\Theta}$ is such that all the matrices $G_{\langle\theta\rangle}, \theta \in \bar{\Theta}$, have only one zero eigenvalue.

Furthermore, there exist $\bar{\mu}>0$ and $\bar{\varepsilon}(\cdot)>0$ such that, for all $\mu>\bar{\mu}$ and for all $\varepsilon \in(0, \bar{\varepsilon}(\mu))$, the regulator (42), (46)-(50), with initial state such that

$$
c^{\prime} \bar{z}_{i}(0)=0, i=1,2, \ldots, n-p
$$

solves the problem.

Notice that the solvability condition above does not depend on $n$ and $p$, and is the same as that presented in [39] for oscillators networks.

Contrary to what has been claimed about condition (24) of Problem 3.1, the necessary and sufficient solvability condition of Problem 4.1 can be given a nice interpretation in terms of the graph of the network (1), (2) and (3). Indeed, it is well known that a topology matrix has got one and only one zero eigenvalue if and only if the corresponding graph is connected (see, e.g., [20]). On the other hand, it is apparent that no consensus problem at all can be solved for networks with unconnected graphs, since the information about the agents' initial conditions cannot be shared over the whole network itself. Consequently, the solvability of Problem 4.1 does not depend on the request of getting a particular agreement function and a particular location of the dominant poles. Put in a different way, Problem 4.1 can be solved if and only if any general consensus problem (without special requirements on the agreement function and on the dominant dynamics) can be solved. In particular, notice that problem solvability is independent of $n$ and $p$.

Let us now illustrate the rationale behind Theorem 4.1. As far as the position of the eigenvalues is concerned, it is quite similar to the one behind Lemma 3.3. First, the fact that $G$ is Laplacian implies that the controlled network has got $n$ eigenvalues at the origin of the complex plane. Then, if we set $\varepsilon=0$ in (48a), the regulator turns out to be nondynamic and to contain multiple derivative actions. In this situation, when $\mu \rightarrow+\infty$, for any $\theta \in \bar{\Theta}$, the closed-loop system has $(n-1)(m-1)$ eigenvalues satisfying (44) and $m-1$ eigenvalues satisfying (45). This is due to the fact that the cascade connection of network and regulator contains $(n-1)(m-1)$ transmission zeros in the locations where the dominant poles have to be placed. Then, by letting $\varepsilon$ be positive and sufficiently small, the previously mentioned eigenvalues still satisfy their respective conditions, the regulator becomes proper, and $(n-p) m$ new eigenvalues appear, which satisfy (45). On the other hand, the fulfilment of point 1 of Problem 4.1 is guaranteed by the sensible choice (48b) for the matrix $\bar{V}$. The details are in the proof.

Similarly to the regulator (23), (25)-(29), (32), (33) solving Problem 3.1, the regulator (42), (46)(50) is actually decentralized, high-gain and with dynamics concentrated in a single transfer function for any agent. However, it is able to solve Problem 4.1 only if its initial state satisfies (51), since, otherwise, the agreement law would not take on the form specified in point 1 of Problem 4.1. Of course, (51) is trivially satisfied by $\bar{z}_{i}(0)=0, i=1,2, \ldots, n-p$. However, it is rather interesting to notice that the regulator initial state can always be set so that (51) is fulfilled and $u(0)=0$. Indeed, on one hand from (49c) it follows that $u(0)=0$ if and only if 


$$
\bar{z}_{1}(0)=\varepsilon^{n-p} \sum_{j \in \mathcal{P} \backslash\{p\}} \bar{e}_{j} \bar{V} y_{j}(0)+\bar{V} y_{p}(0)
$$

On the other hand, (51) is actually satisfied in view of (39), (48b) and Assumption 4.1. Thus, a bumpless control can always be achieved.

We also point out that the regulator (42), (46)-(50) solving Problem 4.1 can be designed according to the following two-step procedure: (i) set $\varepsilon=0$ and find a sufficiently large $\mu$ such that (44), (45) are satisfied; (ii) find a sufficiently small positive $\varepsilon$ such that (44), (45) still hold. The possibility of successfully performing the above steps is guaranteed by Theorem 4.1, as a consequence of its already illustrated rationale.

Finally, it is worth noticing that our solution to Problem 4.1 supplies the controlled network with a nice robustness property. In order to illustrate it, first let $\mathcal{G}$ be the set of all the $m \times m$ symmetric Laplacian matrices with only one zero eigenvalue. Thus, the regulator (42), (46)-(50), besides solving Problem 4.1, is such that points 1 and 2 in it are also satisfied for all the topology matrices belonging to any subset $\overline{\mathcal{G}}$ of $\mathcal{G}$. Indeed, the equations describing our regulator do not depend on the set of considered topology matrices, and the proof of Theorem 4.1 can easily be extended to cope with the new framework. Of course, tuning the parameters $\mu$ and $\varepsilon$ becomes a cumbersome task if $\overline{\mathcal{G}}$ is an infinite set.

As an example, suppose that $\bar{G}_{n}$ is a matrix with only one nonzero eigenvalue which satisfies (36a), (37) for some given $l_{k i}=\bar{l}_{k i}$. Then, all the matrices obtained by substituting $a_{k i} \bar{l}_{k i}$ to $\bar{l}_{k i}$, $a_{i k}=a_{k i}$, in (36a), (37) constitute a meaningful set $\overline{\mathcal{G}}$. Indeed, in the light of (36a), it may though as describing an alteration of the communication apparatuses.

\section{ILLUSTRATIVE EXAMPLES}

The theory presented in the preceding sections is now illustrated by means of a couple of examples, referring to fault-tolerant dominant-pole-placement and to fault-tolerant consensus, respectively.

In both cases, we assume that the network is constituted by four agents with the dynamics of triple integrators, namely, $m=4, n=3$. Furthermore, we consider the two situations where the scalar outputs available to each agent are either 2 or 3 , so that $p=2$ or $p=3$.

\subsection{Example 1: Fault-tolerant Dominant-pole-placement}

We assume here that the network topology matrix is

$$
G=\left[\begin{array}{llll}
1 & 0 & 1 & 6 \\
0 & 3 & 2 & 7 \\
4 & 0 & 5 & 3 \\
0 & 1 & 1 & 7
\end{array}\right]
$$

which satisfies condition (24). Then, we complete the definition of Problem 3.1 by setting $\widehat{\mathcal{L}}_{i}=$ $\{-0.1,-0.2\}, i=1,2,3,4, \alpha=0.4, \beta=1$. By applying Theorem 3.1 with $\mathrm{T}=I_{3}, \varepsilon=0.2, \mu=$ 10 , we get a 4 -th order regulator which solves the problem for $p=2$ and a nondynamic regulator which solves the problem for $p=3$. The maximum relative errors 
Table I. Example 1: Maximum relative errors in the locations of the dominant poles.

\begin{tabular}{ccc}
\hline & $p=2$ & $p=3$ \\
\hline$f_{T}=\emptyset, f_{R}=\emptyset$ & 0.1432 & 0.1087 \\
$f_{T}=\{4\}, f_{R}=\{4\}$ & 0.3514 & 0.3306 \\
$f_{T}=\{3\}, f_{R}=\{1\}$ & 0.0788 & 0.0417 \\
$f_{T}=\{4\}, f_{R}=\{2\}$ & 0.3514 & 0.3306 \\
\hline
\end{tabular}

$$
\max _{i \in\{1,2,3,4\}, j \in\{1,2\}}\left|\frac{\lambda_{i j}-\hat{\lambda}_{i j}}{\hat{\lambda}_{i j}}\right|
$$

in the locations of the dominant poles are reported in Table I, for the nominal and some faulty conditions. As far as the nondominant poles are concerned, the one closest to the imaginary axis has real part -1.3524 in the nominal case and -2.3187 in the faulty cases for $p=2$, and -3.7984 in the nominal case and -1.3544 in the faulty cases for $p=3$.

Moreover, some initial condition responses of the two controlled networks are reported in Figure 1. Specifically, we concentrated on the transients of $x_{1}(t)$ relative to the nominal condition as well as the faulty condition defined by $f_{T}=\{4\}, f_{R}=\{2\}$. We assumed $x_{i}(0)=\mathbf{1}, i=1,2,3$, as network initial condition, and, when $p=2, \hat{z}_{1}(0)=0$ as regulator initial condition. Of course, the drawn responses confirm that the design objectives have been achieved. For both $p=2$ and $p=3$, the transients of $\left[x_{1}\right]_{2}$ and $\left[x_{1}\right]_{4}$ are not significantly affected by the considered fault. On the contrary, the fault influences the transients of $\left[x_{1}\right]_{1}$ and $\left[x_{1}\right]_{3}$. This influence can be reduced by increasing $\mu$ and decreasing $\varepsilon$.

\subsection{Example 2: Fault-tolerant Consensus}

We assume here that the network topology matrix is

$$
G=\left[\begin{array}{cccc}
3 & -1 & -1 & -1 \\
-1 & 2 & -1 & 0 \\
-1 & -1 & 5 & -3 \\
-1 & 0 & -3 & 4
\end{array}\right]
$$

which satisfies Assumption 4.1. Then we complete the definition of Problem 4.1 by letting $\overline{\mathcal{L}}=\{-0.1,-0.2\}, \quad \alpha=0.05, \quad \beta=4, \bar{\Theta}=\{\emptyset,(1,2),(1,3) \cup(1,4),(2,3) \cup(3,4)\}, c=$ $\left[\begin{array}{llll}1 / 2 & 1 / 4 & 1 / 6 & 1 / 12\end{array}\right]^{\prime}$. Straightforward computations show that $G$ and $\bar{\Theta}$ are such that the condition of Theorem 4.1 is satisfied.

By applying Theorem 4.1 with $\varepsilon=0.1, \mu=5$ we get a regulator which solves the problem. The maximum relative errors

$$
\max _{i \in\{1,2,3\}, j \in\{1,2\}}\left|\frac{\lambda_{i j}-\bar{\lambda}_{i j}}{\bar{\lambda}_{i j}}\right|
$$

in the location of the dominant poles are reported in Table II, where the nominal and the faulty conditions are considered. As far as the nondominant poles are concerned, the one closest to the 

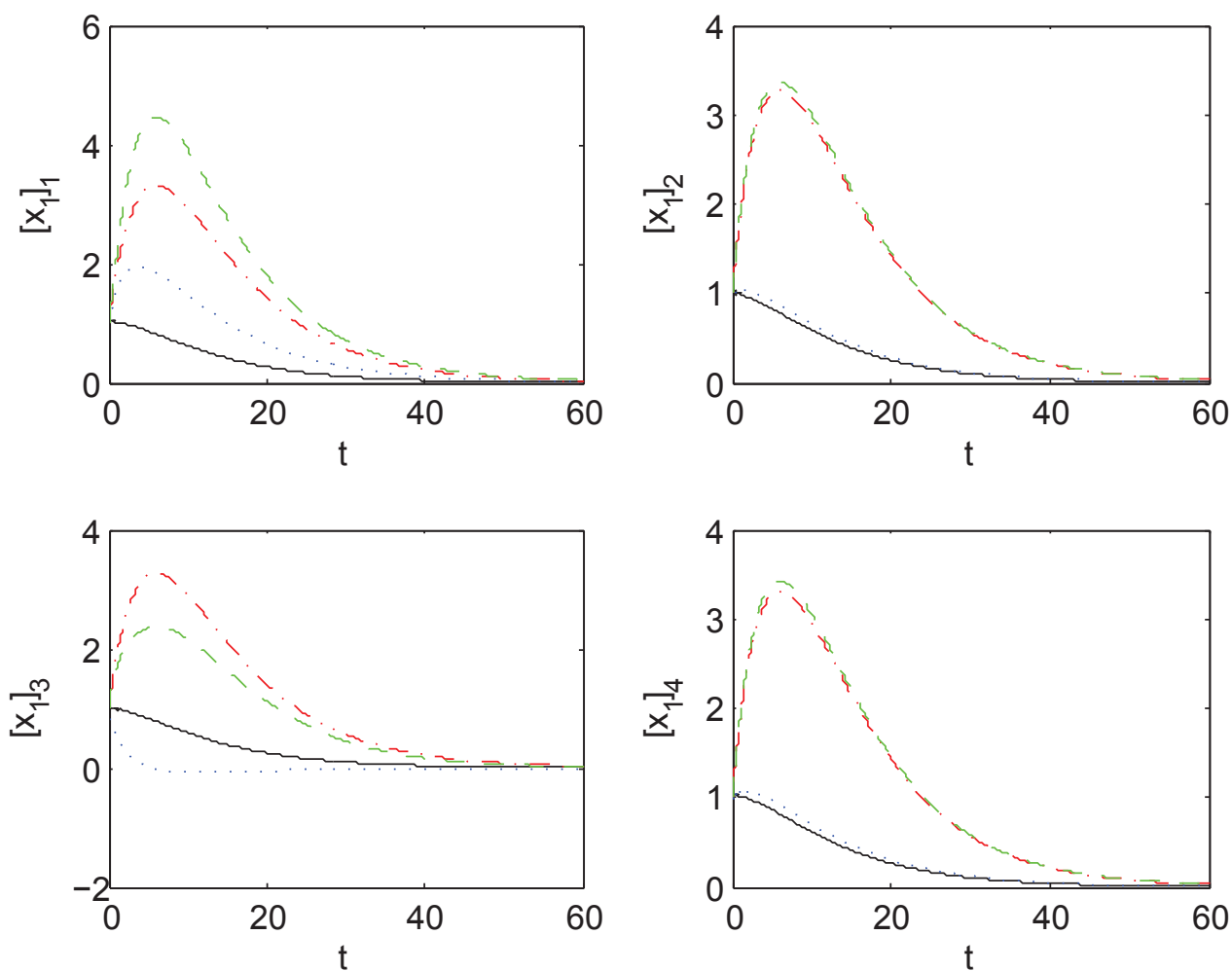

Figure 1. Example 1: Initial condition responses of the variable $x_{1}(t)$ of the controlled network $(p=2$ and nominal condition: black solid; $p=2$ and faulty condition $f_{T}=4, f_{R}=2$ : blue dotted; $p=3$ and nominal condition: red dashdot; $p=3$ and faulty condition $f_{T}=4, f_{R}=2$ : green dashed).

Table II. Example 2: Maximum relative errors in the location of the dominant poles.

\begin{tabular}{ccc}
\hline & $p=2$ & $p=3$ \\
\hline$\theta=\emptyset$ & 0.0277 & 0.0082 \\
$\theta=(1,2)$ & 0.0407 & 0.0215 \\
$\theta=(1,3) \cup(1,4)$ & 0.0481 & 0.0291 \\
$\theta=(2,3) \cup(3,4)$ & 0.0366 & 0.0173 \\
\hline
\end{tabular}

imaginary axis has real part -4.8483 in the nominal case and -4.8465 in the faulty cases for $p=2$, and -48.6312 in the nominal case and -13.8678 in the faulty cases for $p=3$.

Moreover, some initial condition responses are reported in Figures 2 and 3 for $p=2, x_{i}(0)=$ $\left[\begin{array}{llll}1 & 2 & 3 & 4\end{array}\right]^{\prime}, i=1,2,3$, and $\bar{z}_{1}(0)$ as in (52), so as to get a bumpless control.

Note that, in view of (43), the value of $c$ and the considered initial condition, the agreement laws for $x_{i}(t), i=1,2,3$, turn out to be 

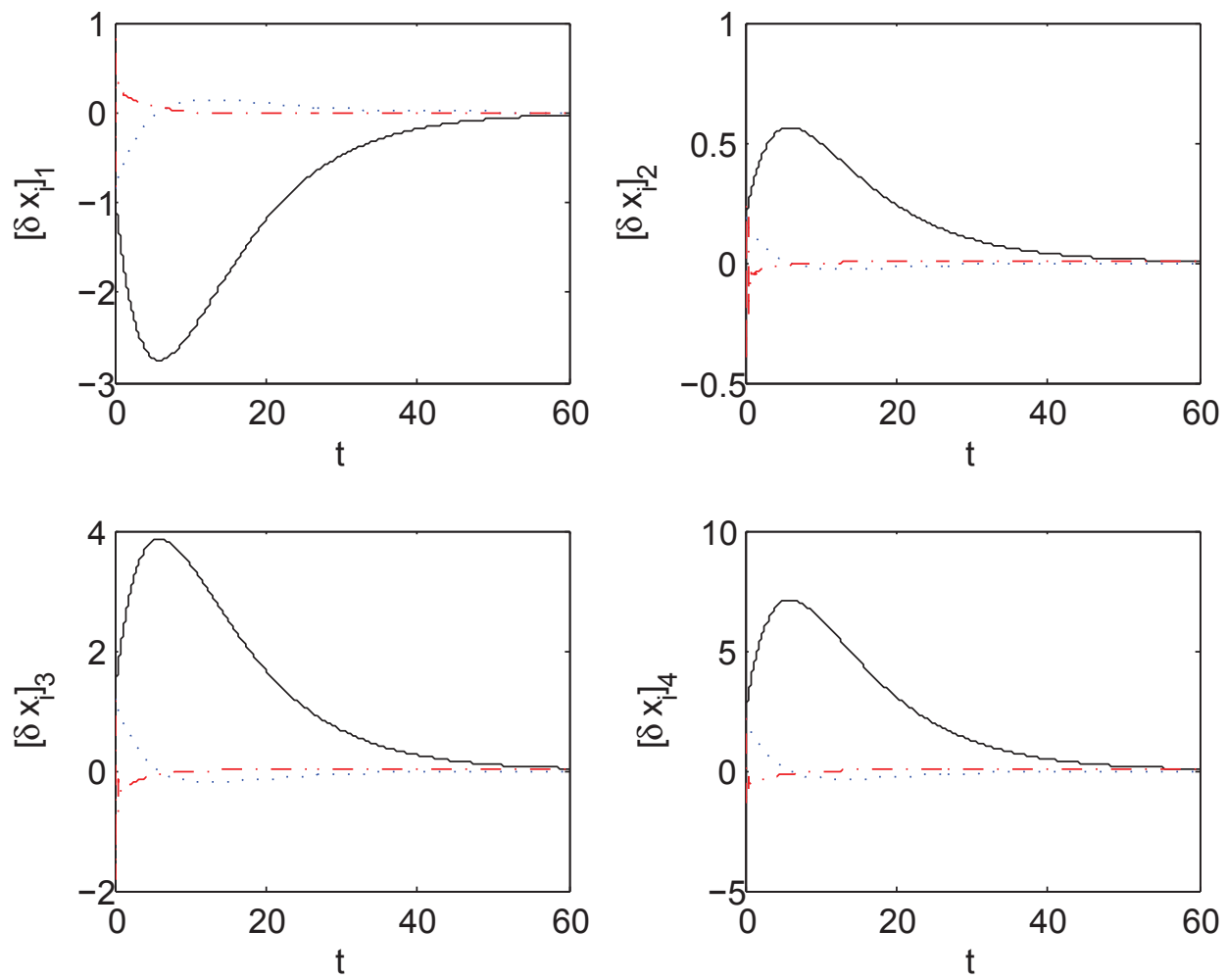

Figure 2. Example 2: Initial condition responses of the variables $\delta x_{i}(t)$ relative to the controlled network in nominal condition $\left(\delta x_{1}(t)\right.$ : black solid; $\delta x_{2}(t)$ : blue dotted; $\delta x_{3}(t)$ : red dashdot).

$$
\begin{gathered}
x_{a g 1}(t):=c^{\prime} \sum_{k=1}^{3} \frac{t^{k-1}}{(k-1) !} x_{k}(0)=\frac{11}{6}\left(1+t+0.5 t^{2}\right) \\
x_{a g 2}(t):=c^{\prime} \sum_{k=2}^{3} \frac{t^{k-2}}{(k-2) !} x_{k}(0)=\frac{11}{6}(1+t) \\
x_{a g 3}(t):=c^{\prime} \sum_{k=3}^{3} \frac{t^{k-3}}{(k-3) !} x_{k}(0)=\frac{11}{6}
\end{gathered}
$$

Specifically, Figure 2 refers to the network nominal condition. It shows the differences $\delta x_{i}(t)$ between the network state variables and the agreement laws, namely $\delta x_{i}(t):=x_{i}(t)-\mathbf{1} x_{a g i}(t)$, $i=1,2,3$. Plainly, the design objectives are fulfilled in this situation.

In order to check that the problem requirements are fulfilled also in the presence of faults, we show in Figure 3 the differences $\delta^{*} x_{i}(t)$ between the state variables of the network in nominal condition and the state variables of the network in the faulty condition $\theta^{*}:=(2,3) \cup(3,4)$, namely $\delta^{*} x_{i}(t):=\left.x_{i}(t)\right|_{\theta=\emptyset}-\left.x_{i}(t)\right|_{\theta=\theta^{*}}$. Apparently, the design objectives are fulfilled in this situation too. 

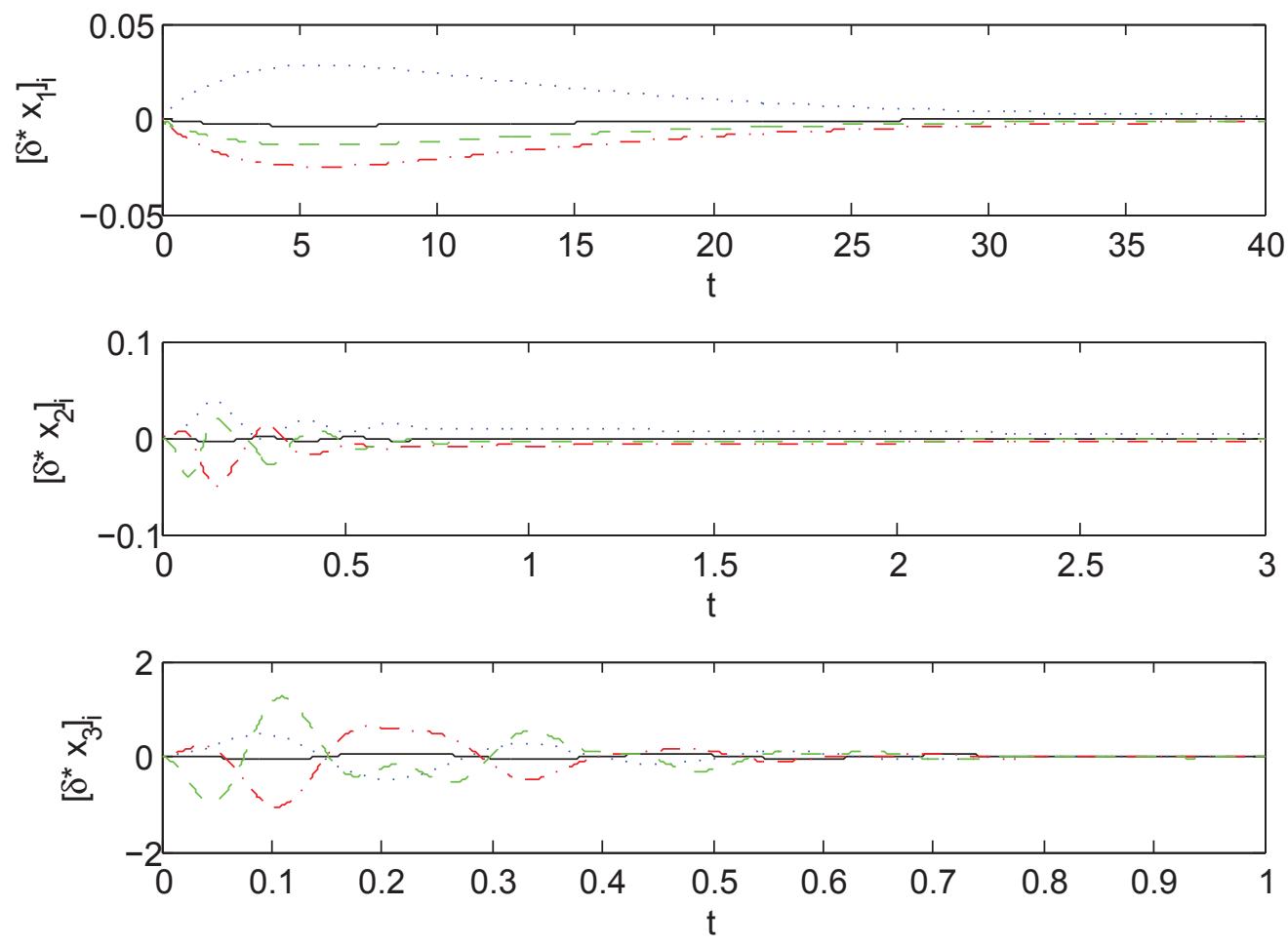

Figure 3. Example 2: Initial condition responses of the variables $\delta^{*} x_{i}(t)$ relative to the controlled network in nominal condition and in the faulty condition $\theta=(2,3) \cup(3,4)\left(\left[\delta^{*} x_{i}(t)\right]_{1}\right.$ : black solid; $\left[\delta^{*} x_{i}(t)\right]_{2}$ : blue dotted; $\left[\delta^{*} x_{i}(t)\right]_{3}$ : red dashdot; $\left[\delta^{*} x_{i}(t)\right]_{4}$ : green dashed).

\section{CONCLUDING REMARKS}

This paper presented a couple of problems concerning the decentralized control of multipleintegrator networks. The first of them is aimed at supplying the controlled network with prespecified real dominant poles, so as to get any desired speed of response. The goal of the second one is achieving a preassigned consensus with arbitrary dynamics. The networks agents considered in the problems are subject to faults of the communication apparatuses, and the regulator must be such that the closed-loop systems are fault-tolerant, that is, the above mentioned properties are not affected by the faults.

For both problems, we gave necessary and sufficient solvability conditions together with explicit formulas for the regulators, which turned out to be high-gain.

The proposed regulators have been shown to be least-order for the first problem. On the contrary, the minimality issue is still to be assessed for the second problem.

Among the other open topics which deserve attention in future works, we cite the extension of the presented results to the cases where the agents' dynamics are not all those of a multiple integrator. Another interesting point concerns the situations where the models of the faults are more general than those adopted here.

\section{REFERENCES}


1. Wu CW, Chua LO. Syncronization in an array of linearly coupled dynamical systems. IEEE Trans. Circuits Syst.-I 1995; 42:430-447.

2. Wu CW, Chua LO. Applications of graph theory to the syncronization in an array of coupled nonlinear oscillators. IEEE Trans. Circuits Syst.-I 1995; 42:494-497.

3. $\mathrm{Wu} \mathrm{CW}$, Chua LO. Applications of Kroneker products to the analysis of systems with uniform linear coupling. IEEE Trans. Circuits Syst.-I 1995; 42:775-778.

4. Fax JA, Murray RM. Information flow and cooperative control of vehicle formations. IEEE Trans. Automat. Control 2004; 49:1465-1476.

5. Ren W, Beard RW. Consensus seeking in multiagent systems under dynamically changing interaction topologies. IEEE Trans. Automat. Control 2005; 50:655-661.

6. Roy S, Saberi A. Static decentralized control of a single-integrator network with markovian sensing topology. Automatica 2005; 41:1867-1877.

7. Olfati-Saber R. Flocking for multi-agent dynamic systems: algorithms and theory. IEEE Trans. Automat. Control 2006; 51:401-420.

8. Xiao F, Wang L, Wang A. Consensus problems in discrete-time multiagent systems with fixed topology. J. Math. Anal. Appl. 2006; 322:587-598.

9. Olfati-Saber R, Fax J, Murray R. Consensus and cooperation in networked multi-agent systems. Proc. of the IEEE 2007; 95:215-233.

10. Ren W. Consensus strategies for cooperative control of vehicle formations. IET Control Theory Appl. 2007; 1:505512.

11. Ren W. Multi-vehicle consensus with a time-varying reference state. Syst. Control Lett. 2007; 56:474-483.

12. Ren W, Moore KL, Chen Y. High-order and model reference consensus algorithms in cooperative control of multivehicle systems. Trans. ASME 2007; 129:678-688.

13. Ren W, Atkins E. Distributed multi-vehicle coordinated control via local information exchange. Int. J. Robust Nonlinear Contr. 2007; 17:1002-1033.

14. Roy S, Saberi A, Herlugson K. A control-theoretic perspective on the design of distributed agreement protocols. Int. J. Robust Nonlinear Contr. 2007; 17:1034-1066.

15. Xiao F, Wang L. Consensus problems for high-dimensional multi-agent systems. IET Control Theory Appl. 2007; 1:830-837.

16. Seo J, Shim H, Back J. Consensus of high-order linear systems using dynamic output feedback compensator: lowgain approach. Automatica 2009; 45:2659-2664.

17. Jiang F, Wang L. Consensus seeking of high-order dynamic multi-agent systems with fixed and switching topologies. Int. J Control 2010; 83:404-420.

18. Li Z, Zhisheng D, Chen G. Consensus of multiagent systems and synchronization of complex networks: a unified viewpoint. IEEE Trans. Circuits Systs. -I 2010; 57:213-224.

19. Ma CQ, Zang JF. Necessary and sufficient conditions for consensusability of linear multi-agent systems. IEEE Trans. Automat. Control 2010; 55:1263-1268.

20. Yu W, Chen G, Ren W, Kurths J, Zheng WX. Distributed high order consensus protocols in multiagents dynamical systems. IEEE Trans. Circuits Syst.-I 2011; 58:1924-1932.

21. Gu G, Marinovici L, Lewis FL. Consensusability of discrete-time dynamic multiagent systems. IEEE Trans. Automat. Control 2012; 57:2085-2089.

22. Roy S, Wan Y, Saberi A, Xue M. Designing linear distributed algorithms with memory for fast convergence. Int. J. Robust Nonlinear Contr. 2012; 22:1691-1702.

23. Abdessameud A, Tayebi A. On consensus algorithms design for double integrator dynamics. Automatica 2013; 49:253-260.

24. Hengster-Movric K, You K, Lewis FL, Xie L. Synchronization of discrete-time multi-agent systems on graphs using Riccati design. Automatica 2013; 49:414-423.

25. Ding L, Han Q, Guo G. Network-based leader-following consensus for distributed multi-agent systems. Automatica 2013; 49:2281-2286.

26. Li Z, Duan Z, Lewis FL. Distributed robust consensus control of multi-agent systems with heterogeneous matching uncertainties. Automatica 2014; 50:883-889.

27. Feng Y, Xu S, Zhang B. Group consensus control for double-integrator dynamic multiagent systems with fixed communication topology. Int. J. Robust Nonlinear Contr. 2014; 24:532-547.

28. Corfmat JP, Morse AS. Stabilization with decentralized feedback control. IEEE Trans. Automat. Control 1973; 18:679-682. 
29. Duan Z, Wang J, Chen G, Huang L. Stability analysis and decentralized control of a class of complex dynamical networks. Automatica 2008; 44:1028-1035.

30. Wan Y, Roy S, Saberi A, Stoorvogel A. The design of multi-lead-compensator for stabilization and pole placement in double-integrator networks. IEEE Trans. Automat. Control 2010; 55:2870-2875.

31. Locatelli A, Schiavoni N. Fault-tolerant stabilization in double-integrator networks. Int. J. Control 2012; 85:16631670.

32. Locatelli A, Schiavoni N. Fault-tolerant stabilization in discrete-time multiple-integrator networks. Proc. American Contr. Conf., Montréal, Québec, Canada, 2012; 1231-1236.

33. Locatelli A, Schiavoni N. Fault-tolerant stabilization in multiple-integrator networks with general information sharing. Proc. Conf. Dec. Contr., Florence, Italy, 2013; 666-671.

34. Locatelli A, Schiavoni N. Fault-tolerant stabilization in discrete-time multiple-integrator networks with general information sharing. Asian J. Control 2014; to appear; .

35. Locatelli A, Schiavoni N. Fault-tolerant pole-placement in double-integrator networks. IEEE Trans. Automat. Control 2012; 57:2912-2917.

36. Locatelli A, Schiavoni N. Fault-tolerant pole-placement in single-integrator networks. Proc. Europ. Contr. Conf., Zürich, Switzerland, 2013; 2945-2950.

37. Su Y, Huang J. Two consensus problems for discrete-time multi-agent systems with switching network topology. Automatica 2012; 48:1988-1997.

38. Locatelli A, Schiavoni N. Fault-tolerant consensus in integrator networks with assignment of agreement law and dynamics. Proc. Conf. Dec. Contr., Florence, Italy, 2013; 672-677.

39. Locatelli A, Schiavoni N. Fault-tolerant consensus in oscillator networks with assignment of agreement laws and dynamics. 22nd Proc. Med. Conf. Contr. Autom., Palermo, Italy, 2014; to appear.

40. Locatelli A, Schiavoni N. Reliable regulation in centralized control systems. Automatica 2009; 45:2673-2677.

41. Locatelli A, Schiavoni N. Fault hiding and reliable regulation in control systems subject to polynomial exogenous signals. Europ. J. Control 2010; 4:389-400.

42. Locatelli A, Schiavoni N. Reliable regulation by high-gain feedback. Proc. 18th Med. Conf. Contr. Autom., Marrakech, Morocco, 2010; 1049-1054.

43. Locatelli A, Schiavoni N. Reliable regulation in decentralized control systems. Int. J. Control 2011; 84:574-583.

44. Corfmat JP, Morse AS. Decentralized control of linear multivariable systems. Automatica 1976; 12:479-495.

45. Wang S, Davison EJ. On the stabilization of decentralized control systems. IEEE Trans. Automat. Control 1973; 18:473-478.

46. Locatelli A, Schiavoni N. A necessary and sufficient condition for the stabilisation of a matrix and its principal submatrices. Linear Algebra Appl. 2012; 436:2311-2314.

47. Kokotovic PV, Khalil HK, O'Reilly J. Singular perturbation methods in control: analysis and design. Academic Press: New York, USA, 1986.

\section{APPENDIX: PROOFS}

\section{Proof of Lemma 3.1}

First assume that $f_{T} \cup f_{R} \subset \mathcal{M}$. Equations (14) of Problem 3.1 reduce to (20) of Problem 3.2 for $f=f_{T} \cup f_{R}$ and $f=\mathcal{M} \backslash\{i\}, i \in f_{T} \cup f_{R}$, respectively. Viceversa, (20) of Problem 3.2 reduces to (14a) of Problem 3.1 if $f_{T} \cup f_{R}=f$. Secondly, assume that $f_{T} \cup f_{R}=\mathcal{M}$. In this case, (14b) of Problem 3.1 coincides with (20) of Problem 3.2 for $f=\mathcal{M} \backslash\{i\}, i \in \mathcal{M}$. Furthermore, conditions (15)-(18) coincide with (21), (22).

\section{Proof of Lemma 3.2}

Assume that a regulator $R(s)$ given by (4b)-(4d), (5) solves Problem 3.2. Then, all the roots of (20), $f \subset \mathcal{M}$, have negative real part. Hence, the coefficients of the terms with highest and lowest degree of $\operatorname{det}\left(Q_{(f)}(s)\right)$ have the same sign. The former originates from $\operatorname{det}\left(s^{n} D_{(f)}(s)\right)$ and is equal to one, since the polynomials $d_{i}(s), i \in \mathcal{M}$, are monic. Thus, the latter must be positive, that is, 


$$
\operatorname{det}\left(-N_{1(f)}(0) G_{(f)}\right)=\operatorname{det}\left(G_{(f)}\right) \sum_{i \in \mathcal{M} \backslash f}\left(-n_{1 i}(0)\right)>0, \forall f \subset \mathcal{M}
$$

Consider now the particular case where $f=\mathcal{M} \backslash\{i\}, i \in \mathcal{M}$. Then, condition (53) becomes

$$
-g_{i, i} n_{1 i}(0)>0, \forall i \in \mathcal{M}
$$

which means that $g_{i, i}$ and $n_{1 i}(0)$ have opposite signs. Hence, (53), (54) yield

$$
\operatorname{det}\left(G_{(f)}\right) \sum_{i \in \mathcal{M} \backslash f} g_{i, i}>0, \forall f \subset \mathcal{M}
$$

which obviously supplies (24), in view of (23).

\section{Proof of Lemma 3.3}

Recall (31). Moreover, let

$$
A:=\left[\begin{array}{ccccc}
-1 & 1 & 0 & \cdots & 0 \\
0 & -1 & 1 & \cdots & 0 \\
\vdots & \vdots & \vdots & \ddots & \vdots \\
0 & 0 & 0 & \cdots & -1
\end{array}\right] \in \mathrm{R}^{(n-p) \times(n-p)}, B:=\left[\begin{array}{c}
0 \\
0 \\
\vdots \\
1
\end{array}\right] \in \mathrm{R}^{n-p}, C^{\prime}:=\left[\begin{array}{c}
1 \\
0 \\
\vdots \\
0
\end{array}\right] \in \mathrm{R}^{n-p}
$$

Now, for any $f \subset \mathcal{M}$, consider the $(2 n-p)\left(m-\kappa_{f}\right)$-th order system

$$
\begin{gathered}
\dot{x}_{i(f)}(t)=x_{i+1(f)}(t), i \in \mathcal{N} \backslash\{n\} \\
\dot{x}_{n(f)}(t)=u_{(f)}(t) \\
\varepsilon \dot{z}_{i}(t)=A z_{i}(t)+B \sum_{j=p}^{n}\left[\widehat{E}_{j(f)}\right]_{i} \widehat{V}_{(f)} G_{(f)} x_{j(f)}(t), i \in \mathcal{M} \backslash f \\
w_{(\mathcal{M} \backslash\{i\})}(t)=[w(t)]_{i}=\mu\left(C z_{i}(t)+\sum_{j \in \mathcal{P} \backslash\{p\}}\left[\widehat{E}_{j(f)}\right]_{i} \widehat{V}_{(f)} G_{(f)} x_{j(f)}(t)\right), i \in \mathcal{M} \backslash f
\end{gathered}
$$

where $w(t) \in \mathrm{R}^{m}$.

Simple, though cumbersome, computations show that the transfer function of system (55) from $u_{(f)}(t)$ to $w_{(f)}(t)$ is exactly the function $\widehat{L}_{(f)}(s)$ given in (31). Hence, the roots of (20) do coincide with the eigenvalues of system (55), after setting $u_{(f)}(t)=w_{(f)}(t)$. The dynamical matrix of this system is 


$$
\widehat{F}(f):=\left[\begin{array}{cccccccc}
0 & I_{m-\kappa_{f}} & \cdots & 0 & 0 & \cdots & 0 & 0 \\
0 & 0 & \cdots & 0 & 0 & \cdots & 0 & 0 \\
\vdots & \vdots & \ddots & \vdots & \vdots & \ddots & \vdots & \vdots \\
0 & 0 & \cdots & 0 & 0 & \cdots & I_{m-\kappa_{f}} & 0 \\
\mu \widehat{\widehat{E}}_{1}(f) & \mu \widehat{\widehat{E}}_{2}(f) & \cdots & \mu \widehat{\widehat{E}}_{p-1}(f) & 0 & \cdots & 0 & \mu \widehat{C}(f) \\
0 & 0 & \cdots & 0 & \varepsilon^{-1} \widetilde{E}_{p}(f) & \cdots & \varepsilon^{-1} \widetilde{E}_{n}(f) & \varepsilon^{-1} \widehat{A}(f)
\end{array}\right]
$$

where

$$
\begin{gathered}
\widehat{A}(f):=\operatorname{diag}\{\underbrace{A, A, \ldots, A}_{m-\kappa_{f} \text { blocks }}\}, \widehat{B}(f):=\operatorname{diag}\{\underbrace{B, B, \ldots, B}_{m-\kappa_{f} \text { blocks }}\}, \widehat{C}(f):=\operatorname{diag}\{\underbrace{C, C, \ldots, C}_{m-\kappa_{f} \text { blocks }}\} \\
\widehat{\widehat{E}}_{i}(f):=\widehat{V}(f) \widehat{E}_{i(f)} G_{(f)}, i \in \mathcal{P} \backslash\{p\} \\
\widetilde{E}_{i}(f):=\widehat{B}(f) \widehat{V}(f) \widehat{E}_{i(f)} G_{(f)}, i=p, p+1, \ldots, n
\end{gathered}
$$

Now, remind that the order of each local regulator is $\rho_{i}=n-p$. Thus, the number of the eigenvalues which have to comply with (22) is $(1+n-p)\left(m-\kappa_{f}\right)$.

By applying standard singular perturbation theory [47], when $\varepsilon \rightarrow 0, \quad(n-p)\left(m-\kappa_{f}\right)$ eigenvalues of (56) tend to the eigenvalues of $\varepsilon^{-1} \widehat{A}(f)$, which are all at $\varepsilon^{-1}$. Hence, if $\varepsilon$ is positive and sufficiently small, they satisfy (22).

The remaining $n\left(m-\kappa_{f}\right)$ eigenvalues of $\widehat{F}(f)$ tend to the eigenvalues of

$$
\widehat{F}_{s}(f):=\left[\begin{array}{ccccccc}
0 & I_{m-\kappa_{f}} & \cdots & 0 & 0 & \cdots & 0 \\
0 & 0 & \cdots & 0 & 0 & \cdots & 0 \\
\vdots & \vdots & \ddots & \vdots & \vdots & \ddots & \vdots \\
0 & 0 & \cdots & 0 & 0 & \cdots & I_{m-\kappa_{f}} \\
\mu \widehat{\widehat{E}}_{1}(f) & \mu \widehat{\widehat{E}}_{2}(f) & \cdots & \mu \widehat{\widehat{E}}_{p-1}(f) & \mu \widehat{\widehat{E}}_{p}(f) & \cdots & \mu \widehat{\widehat{E}}_{n}(f)
\end{array}\right]
$$

where

$$
\begin{gathered}
\widehat{\widehat{E}}_{i}(f):=-\widehat{C}(f) \widehat{A}(f)^{-1} \widetilde{\widehat{E}}_{i}(f)=-\widehat{C}(f) \widehat{A}(f)^{-1} \widehat{B}(f) \widehat{V}(f) \widehat{E}_{i(f)} G_{(f)}= \\
=\widehat{V}(f) \widehat{E}_{i(f)} G_{(f)}, i=p, p+1, \ldots, n
\end{gathered}
$$

since $\widehat{C}(f) \widehat{A}(f)^{-1} \widehat{B}(f)=-I_{m-\kappa_{f}}$.

By again applying standard singular perturbation theory, when $\mu \rightarrow+\infty, m-\kappa_{f}$ eigenvalues of (57) tend to the eigenvalues of $\mu \widehat{\widehat{E}}_{n}(f)=\mu \widehat{V}_{(f)} \widehat{E}_{n(f)} G_{(f)}=\mu \widehat{V}_{(f)} G_{(f)}$, in view of (25) and (26a). Since the matrices $\widehat{V}_{(f)} G_{(f)}$ are Hurwitz by assumption, for $\mu$ sufficiently large, these eigenvalues satisfy (22).

The remaining $(n-1)\left(m-\kappa_{f}\right)$ eigenvalues of $\widehat{F}_{s}(f)$ tend to the eigenvalues of 


$$
\widehat{F}_{s s}(f):=\left[\begin{array}{cccc}
0 & I_{m-\kappa_{f}} & \cdots & 0 \\
0 & 0 & \cdots & 0 \\
\vdots & \vdots & \ddots & \vdots \\
0 & 0 & \cdots & I_{m-\kappa_{f}} \\
-\widehat{E}_{1}(f) & -\widehat{E}_{2}(f) & \cdots & -\widehat{E}_{n-1}(f)
\end{array}\right]
$$

whose characteristic polynomial is

$$
\operatorname{det}\left(\lambda I_{(n-1)\left(m-\kappa_{f}\right)}-\widehat{F}_{s s}(f)\right)=\prod_{i=1}^{m-\kappa_{f}}\left(\sum_{j \in \mathcal{N}} \hat{e}_{i j} \lambda^{j-1}\right)
$$

Hence, in view of (25), the eigenvalues of $\widehat{F}_{s s}(f)$ satisfy (21).

\section{Proof of Lemma 3.4}

The proof can be performed along the same steps as those of the proof of Theorem 2.1 in [46], after substituting matrix $G_{D}$ there with matrix $\widetilde{G}$.

\section{Proof of Theorem 3.1}

Necessity follows from Lemmas 3.1 and 3.2. Sufficiency follows from Lemmas 3.1, 3.3 and 3.4.

\section{Proof of Theorem 4.1}

As for necessity, we assume by contradiction that there exists $\theta^{*} \in \bar{\Theta}$ such that the (symmetric) matrix $G_{\left\langle\theta^{*}\right\rangle}$ has more than one zero eigenvalue. Thus, for $\theta=\theta^{*}$, the network is composed by (at least) two completely separated subnetworks (see, e.g., [20]). Hence, no agreement law can be reached, and point 1 of Problem 4.1 is violated.

As for sufficiency, we assume $\theta \in \bar{\Theta}$. Then, as a first step, we show that the regulator supplies the controlled network with the required eigenvalues (point 2 of Problem 4.1), and, as a second one, we show that the asymptotic behavior is the one called for (point 1 of Problem 4.1).

Let us begin by studying point 2 , that is, the eigenvalues of the controlled network, described by (1), (39), (42), (46)-(50).

Let $T(\theta) \in \mathrm{R}^{m \times m}$ be a nonsingular matrix such that

$$
\bar{V} G_{\langle\theta\rangle}=T(\theta)^{-1} S(\theta) T(\theta), S(\theta):=\left[\begin{array}{cc}
\bar{S}(\theta) & 0 \\
0 & 0
\end{array}\right] \in \mathrm{R}^{m \times m}
$$

where $\bar{S}(\theta) \in \mathrm{R}^{(m-1) \times(m-1)}$ is nonsingular, since $G_{\langle\theta\rangle}$ has got exactly one zero eigenvalue and $\bar{V}$ is nonsingular. Furthermore, it is Hurwitz, because the nonzero eigenvalues of $\bar{V} G_{\langle\theta\rangle}$ have negative real parts in view of Assumption 4.1, (42), (48b) and the Gershgorin theorem.

Furthermore, let 


$$
\begin{aligned}
& \bar{x}_{i}:=T(\theta) x_{i}:=\left[\begin{array}{cc}
\bar{x}_{i a}^{\prime} & \bar{x}_{i b}
\end{array}\right]^{\prime}, \bar{x}_{i a} \in \mathrm{R}^{m-1}, \bar{x}_{i b} \in \mathrm{R}, i \in \mathcal{N} \\
& \overline{\bar{z}}_{i}:=T(\theta) \bar{z}_{i}:=\left[\begin{array}{cc}
\overline{\bar{z}}_{i a}^{\prime} & \overline{\bar{z}}_{i b}
\end{array}\right]^{\prime}, \overline{\bar{z}}_{i a} \in \mathrm{R}^{m-1}, \overline{\bar{z}}_{i b} \in \mathrm{R}, i=1,2, \ldots, n-p \\
& \eta_{1}:=\left[\begin{array}{cccccccc}
\bar{x}_{1 a}^{\prime} & \bar{x}_{2 a}^{\prime} & \cdots & \bar{x}_{n a}^{\prime} & \overline{\bar{z}}_{1 a}^{\prime} & \overline{\bar{z}}_{2 a}^{\prime} & \cdots & \overline{\bar{z}}_{n-p a}^{\prime}
\end{array}\right]^{\prime} \in \mathrm{R}^{(2 n-p)(m-1)} \\
& \eta_{2}:=\left[\begin{array}{llll}
\bar{x}_{1 b} & \bar{x}_{2 b} & \cdots & \bar{x}_{n b}
\end{array}\right]^{\prime} \in \mathrm{R}^{n} \\
& \eta_{3}:=\left[\begin{array}{llll}
\overline{\bar{z}}_{1 b} & \overline{\bar{z}}_{2 b} & \cdots & \overline{\bar{z}}_{n-p b}
\end{array}\right]^{\prime} \in \mathrm{R}^{n-p}
\end{aligned}
$$

Trivial, though cumbersome, computations show that

$$
\eta:=\left[\begin{array}{lll}
\eta_{1}^{\prime} & \eta_{2}^{\prime} & \eta_{3}^{\prime}
\end{array}\right]^{\prime}=R \bar{T}(\theta)\left[\begin{array}{cccccccc}
x_{1}^{\prime} & x_{2}^{\prime} & \cdots & x_{n}^{\prime} & \bar{z}_{1}^{\prime} & \bar{z}_{2}^{\prime} & \cdots & \bar{z}_{n-p}^{\prime}
\end{array}\right]^{\prime}
$$

where

$$
\begin{gathered}
\bar{T}(\theta):=\operatorname{diag}\{\underbrace{T(\theta), T(\theta), \ldots, T(\theta)}_{2 n-p \text { blocks }}\} \in \mathrm{R}^{(2 n-p) m \times(2 n-p) m} \\
R:=\left[\operatorname{diag}\{\underbrace{R_{a}, R_{a}, \ldots, R_{a}}_{2 n-p \text { blocks }}\}\right] \in \mathrm{R}^{(2 n-p) m \times(2 n-p) m} \\
\left.\operatorname{diag}\{\underbrace{R_{b}, R_{b}, \ldots, R_{b}}_{2 n-p \text { blocks }}\}\right] \\
R_{a}:=\left[\begin{array}{ll}
I_{m-1} & 0] \in \mathrm{R}^{(m-1) \times m}, R_{b}:=\left[\begin{array}{ll}
0 & 1
\end{array}\right] \in \mathrm{R}^{1 \times m}
\end{array}\right.
\end{gathered}
$$

Consequently, the controlled network (1), (39), (42), (46)-(50) is also described by the equation

$$
\dot{\eta}(t)=\bar{F}(\theta) \eta(t)
$$

where

$$
\bar{F}(\theta):=\left[\begin{array}{ccc}
\bar{F}_{1}(\theta) & 0 & 0 \\
0 & \bar{F}_{2} & \bar{F}_{23} \\
0 & 0 & \bar{F}_{3}
\end{array}\right] \in \mathrm{R}^{(2 n-p) m \times(2 n-p) m}
$$




$$
\begin{aligned}
& \bar{F}_{1}(\theta):=\left[\begin{array}{cc}
\bar{F}_{1 x}(\theta) & \bar{F}_{1 x z} \\
\bar{F}_{1 z x}(\theta) & \bar{F}_{1 z}
\end{array}\right] \in \mathrm{R}^{(2 n-p)(m-1) \times(2 n-p)(m-1)} \\
& \bar{F}_{1 x}(\theta):= \\
& :=\left[\begin{array}{ccccccc}
0 & I_{m-1} & \cdots & 0 & 0 & \cdots & 0 \\
0 & 0 & \cdots & 0 & 0 & \cdots & 0 \\
\vdots & \vdots & \ddots & \vdots & \vdots & \ddots & \vdots \\
0 & 0 & \cdots & 0 & 0 & \cdots & I_{m-1} \\
\mu \bar{e}_{1} \bar{S}(\theta) & \mu \bar{e}_{2} \bar{S}(\theta) & \cdots & \mu \bar{e}_{p-1} \bar{S}(\theta) & \mu \varepsilon^{-(n-p)} \bar{S}(\theta) & \cdots & 0
\end{array}\right] \in \mathrm{R}^{n(m-1) \times n(m-1)} \\
& \bar{F}_{1 z x}(\theta):=\left[\begin{array}{ccccccc}
0 & 0 & \cdots & 0 & \varepsilon^{-1} \bar{h}_{1} \bar{S}(\theta) & \cdots & 0 \\
0 & 0 & \cdots & 0 & \varepsilon^{-1} \bar{h}_{2} \bar{S}(\theta) & \cdots & 0 \\
\vdots & \vdots & \ddots & \vdots & \vdots & \ddots & \vdots \\
0 & 0 & \cdots & 0 & \varepsilon^{-1} \bar{h}_{n-p-1} \bar{S}(\theta) & \cdots & 0 \\
0 & 0 & \cdots & 0 & \varepsilon^{-1} \bar{h}_{n-p} \bar{S}(\theta) & \cdots & 0
\end{array}\right] \in \mathrm{R}^{(n-p)(m-1) \times n(m-1)} \\
& \bar{F}_{1 x z}:=\left[\begin{array}{ccccc}
0 & 0 & 0 & \cdots & 0 \\
\vdots & \vdots & \vdots & \ddots & \vdots \\
0 & 0 & 0 & \cdots & 0 \\
-\mu \varepsilon^{-(n-p)} I_{m-1} & 0 & 0 & \cdots & 0
\end{array}\right] \in \mathrm{R}^{n(m-1) \times(n-p)(m-1)} \\
& \bar{F}_{1 z}:=\left[\begin{array}{ccccc}
-\varepsilon^{-1} I_{m-1} & \varepsilon^{-1} I_{m-1} & 0 & \cdots & 0 \\
0 & -\varepsilon^{-1} I_{m-1} & \varepsilon^{-1} I_{m-1} & \cdots & 0 \\
\vdots & \vdots & \vdots & \ddots & \vdots \\
0 & 0 & 0 & \cdots & \varepsilon^{-1} I_{m-1} \\
0 & 0 & 0 & \cdots & -\varepsilon^{-1} I_{m-1}
\end{array}\right] \in \mathrm{R}^{(n-p)(m-1) \times(n-p)(m-1)}
\end{aligned}
$$




$$
\begin{aligned}
& \bar{F}_{2}:=\left[\begin{array}{cccccc}
0 & 1 & 0 & \cdots & 0 & 0 \\
0 & 0 & 1 & \cdots & 0 & 0 \\
\vdots & \vdots & \vdots & \ddots & \vdots & \vdots \\
0 & 0 & 0 & \cdots & 0 & 1 \\
0 & 0 & 0 & \cdots & 0 & 0
\end{array}\right] \in \mathrm{R}^{n \times n} \\
& \bar{F}_{23}:=\left[\begin{array}{ccccc}
0 & 0 & 0 & \cdots & 0 \\
0 & 0 & 0 & \cdots & 0 \\
\vdots & \vdots & \vdots & \ddots & \vdots \\
0 & 0 & 0 & \cdots & 0 \\
-\mu \varepsilon^{-(n-p)} & 0 & 0 & \cdots & 0
\end{array}\right] \in \mathrm{R}^{n \times(n-p)} \\
& \bar{F}_{3}:=\left[\begin{array}{ccccc}
-\varepsilon^{-1} & \varepsilon^{-1} & 0 & \cdots & 0 \\
0 & -\varepsilon^{-1} & \varepsilon^{-1} & \cdots & 0 \\
\vdots & \vdots & \vdots & \ddots & \vdots \\
0 & 0 & 0 & \cdots & \varepsilon^{-1} \\
0 & 0 & 0 & \cdots & -\varepsilon^{-1}
\end{array}\right] \in \mathrm{R}^{(n-p) \times(n-p)}
\end{aligned}
$$

Hence, the eigenvalues of $\bar{F}(\theta)$ are those of $\bar{F}_{1}(\theta), \bar{F}_{2}$ and $\bar{F}_{3}$. The $n$ eigenvalues of $\bar{F}_{2}$ are all at the origin of the complex plane. As a matter of fact, they are the network fixed modes mentioned in commenting the statement of Problem 4.1. The $n-p$ eigenvalues of $\bar{F}_{3}$ are all at $-\varepsilon^{-1}$. As far as matrix $\bar{F}_{1}(\theta)$ is concerned, notice that it is the dynamical matrix of the $(2 n-p)(m-1)$-order system

$$
\begin{gathered}
\dot{\bar{x}}_{i a}(t)=\dot{\bar{x}}_{i+1 a}(t), i \in \mathcal{N} \backslash\{n\} \\
\dot{\bar{x}}_{n a}(t)=\mu\left\{\sum_{j \in \mathcal{P} \backslash\{p\}} \bar{e}_{j} \bar{S}(\theta) \bar{x}_{j a}(t)+\varepsilon^{-(n-p)} \bar{S}(\theta) \bar{x}_{p a}(t)-\varepsilon^{-(n-p)} \overline{\bar{z}}_{1 a}(t)\right\} \\
\varepsilon \dot{\overline{\bar{z}}}_{i a}(t)=-\overline{\bar{z}}_{i a}(t)+\overline{\bar{z}}_{i+1 a}(t)+\bar{h}_{i} \bar{S}(\theta) \bar{x}_{p a}(t), i=1,2, \ldots, n-p-1 \\
\varepsilon \dot{\overline{\bar{z}}}_{n-p a}(t)=-\overline{\bar{z}}_{n-p a}(t)+\bar{h}_{n-p} \bar{S}(\theta) \bar{x}_{p a}(t)
\end{gathered}
$$

where the coefficients $\bar{h}_{i}$ are defined through the identity (50). This system has the same structure as the one in (30). Consequently, by following the same line of reasoning as in the proof ol Lemma 3.3, it can be proved that it has: (i) $(n-p)(m-1)$ eigenvalues that, when $\varepsilon \rightarrow 0$, tend to $-\varepsilon^{-1}$; (ii) $m-1$ eigenvalues that, when $\mu \rightarrow+\infty$, tend to the eigenvalues of $\mu \bar{S}(\theta)$; their real parts tend to $-\infty$ since $\bar{S}(\theta)$ is Hurwitz; (iii) $(n-1)(m-1)$ eigenvalues that, when $\mu \rightarrow+\infty$, tend to the eigenvalues of 


$$
\bar{F}_{s s}:=\left[\begin{array}{cccc}
0 & I_{m-1} & \cdots & 0 \\
0 & 0 & \cdots & 0 \\
\vdots & \vdots & \ddots & \vdots \\
0 & 0 & \cdots & I_{m-1} \\
-\bar{e}_{1} I_{m-1} & -\bar{e}_{2} I_{m-1} & \cdots & -\bar{e}_{n-1} I_{m-1}
\end{array}\right]
$$

whose characteristic polynomial is

$$
\operatorname{det}\left(\lambda I_{(n-1)(m-1)}-\bar{F}_{s s}\right)=\left(\sum_{j \in \mathcal{N}} \bar{e}_{j} \lambda^{j-1}\right)^{m-1}
$$

since, in view of (46), $\bar{e}_{n}=1$.

In conclusion, for $\varepsilon$ positive and sufficiently small and $\mu$ sufficiently large, matrix $\bar{F}(\theta)$, besides having $n$ eigenvalus at $s=0$, has: (i) $m-1+(n-p) m$ eigenvalues which fulfil (45), since $\rho_{i}=n-p, i \in \mathcal{M}$; (ii) $(n-1)(m-1)$ eigenvalues which fulfil (44). Hence, point 2 is satisfied.

Let us consider now point 1 , that is, the asymptotic behavior of the controlled network, described by (1), (39), (42), (46)-(50).

Notice that the last column of $T(\theta)^{-1}$ can be taken equal to 1 . Thus, the last row of $T(\theta)$, to be denoted by $\psi^{\prime}$, has to satisfy the conditions

$$
\psi^{\prime} \mathbf{1}=1, \psi^{\prime} \bar{V} G_{\langle\theta\rangle}=0
$$

Hence, by recalling that $G_{\langle\theta\rangle}$ is symmetric, it turns out that

$$
\psi=c
$$

Consequently, simple computations show that, in view of (58b), (58e), (63), condition (51) is equivalent to

$$
\eta_{3}(0)=\left[\begin{array}{c}
c^{\prime} \bar{z}_{1}(0) \\
c^{\prime} \bar{z}_{2}(0) \\
\vdots \\
c^{\prime} \bar{z}_{n-p}(0)
\end{array}\right]=0
$$

Thus, in view of (60)-(62), the solution of (59) is given by

$$
\begin{gathered}
\eta_{1}(t)=e^{\bar{F}_{1}(\theta) t} \eta_{1}(0), t \geq 0 \\
\eta_{2}(t)=e^{\bar{F}_{2} t} \eta_{2}(0), t \geq 0 \\
\eta_{3}(t)=0, t \geq 0
\end{gathered}
$$

Since it has already been proved that $\bar{F}_{1}(\theta)$ is Hurwitz, then the solution of (65a) is such that $\lim _{t \rightarrow+\infty} \eta_{1}(t)=0$. On the other hand, in view of (62a), the solution of (65b) is 


$$
\left[\eta_{2}(t)\right]_{i}=\sum_{k=0}^{n-i} \frac{t^{k}}{k !}\left[\eta_{2}(0)\right]_{i+k}, t \geq 0, i \in \mathcal{N}
$$

Now, observe that, in view of (58a), (58d), (63),

$$
\eta_{2}(t)=\left[\begin{array}{c}
c^{\prime} x_{1}(t) \\
c^{\prime} x_{2}(t) \\
\vdots \\
c^{\prime} x_{n}(t)
\end{array}\right]
$$

Since $\eta_{1}(t)$ asymptotically vanishes, then $\lim _{t \rightarrow+\infty} \bar{x}_{i a}(t)=0, i \in \mathcal{N}$, in view of (58c). Furthermore, since $x_{i}(t)-T(\theta)^{-1} \bar{x}_{i}(t)=0$, in view of (58a), (58d),

$$
0=\lim _{t \rightarrow+\infty}\left(x_{i}(t)-T(\theta)^{-1} \bar{x}_{i}(t)\right)=\lim _{t \rightarrow+\infty}\left(x_{i}(t)-\mathbf{1} \bar{x}_{i b}(t)\right)=\lim _{t \rightarrow+\infty}\left(x_{i}(t)-\mathbf{1}\left[\eta_{2}(t)\right]_{i}\right), i \in \mathcal{N}
$$

Finally, in view of (66), (67),

$$
\lim _{t \rightarrow+\infty}\left(x_{i}(t)-\mathbf{1} \sum_{k=0}^{n-i} \frac{t^{k}}{k !} c^{\prime} x_{i+k}(0)\right)=\lim _{t \rightarrow+\infty}\left(x_{i}(t)-\mathbf{1} c^{\prime} \sum_{k=i}^{n} \frac{t^{k-i}}{(k-i) !} x_{k}(0)\right)=0, i \in \mathcal{N}
$$

which coincides with (43), so that point 1 is satisfied.

Thus, the theorem is proved. 